TRANSACTIONS OF THE

AMERICAN MATHEMATICAL SOCIETY

Volume 363, Number 4, April 2011, Pages 2143-2170

S 0002-9947(2010)05185-2

Article electronically published on November 16, 2010

\title{
TOPOLOGICAL INVARIANTS OF PIECEWISE HEREDITARY ALGEBRAS
}

\author{
PATRICK LE MEUR
}

\begin{abstract}
We investigate the Galois coverings of piecewise algebras and more particularly their behaviour under derived equivalences. Under a technical assumption which is satisfied if the algebra is derived equivalent to a hereditary algebra, we prove that there exists a universal Galois covering whose group of automorphisms is free and depends only on the derived category of the algebra. As a corollary, we prove that the algebra is simply connected if and only if its first Hochschild cohomology vanishes.
\end{abstract}

\section{INTRODUCTION}

Let $k$ be an algebraically closed field and $A$ a basic finite dimensional $k$-algebra (or, simply, an algebra). The representation theory studies the category $\bmod A$ of finite dimensional (right) $A$-modules and also its bounded derived category $\mathcal{D}^{b}(\bmod A)$. From this point of view, some classes of algebras play an important role: The hereditary algebras, that is, path algebras $k Q$ of finite quivers $Q$ with no oriented cycles; the tilted algebras, that is, of the form $\operatorname{End}_{k Q}(T)$, where $T$ is a tilting $k Q$-module; and, more generally, the piecewise hereditary algebras, that is, the algebras $A$ such that $\mathcal{D}^{b}(\bmod A)$ is triangle equivalent to $\mathcal{D}^{b}(\mathcal{H})$ where $\mathcal{H}$ is a Hom-finite hereditary abelian category with split idempotents (if $\mathcal{H}=\bmod k Q$, then $A$ is called piecewise hereditary of type $Q$ ). Tilted algebras are particularly well understood (see 2, 43, 44, for instance). Piecewise hereditary algebras have also been deeply investigated. It was proved in [22] (see also [23] and [24]) that the class of connected piecewise hereditary algebras is divided into two subclasses: that of algebras derived equivalent to some path algebra, and that of algebras $A$ such that $\mathcal{D}^{b}(\bmod A) \simeq \mathcal{D}^{b}(\operatorname{coh} \mathbb{X})$ with $\mathbb{X}$ a weighted projective line $(18)$. Also, the Morita theory of Rickard for derived equivalences ([39]) has a nice behaviour for piecewise hereditary algebras. Indeed, it was proved in 26 that if $A$ is derived equivalent to a hereditary algebra $H$, then $A$ is an iterated tilted algebra of $H$. This result was extended in 25] to the setting of all piecewise hereditary algebras (see also 36 for the case of algebras derived equivalent to hereditary abelian categories with tilting complexes). Finally, it was proved in 28 that an algebra is piecewise hereditary if and only if its strong global dimension is finite in the sense of [45] (the tame case was treated in [6]).

The piecewise hereditary algebras arise in many areas of representation theory. For example, the cluster category $C_{A}$ of a piecewise hereditary algebra $A$ was

Received by the editors March 6, 2009 and, in revised form, July 30, 2009.

2010 Mathematics Subject Classification. Primary 16G10; Secondary 16G60, 16E35, 16E40.

(C)2010 American Mathematical Society

Reverts to public domain 28 years from publication 
introduced in [8], 10] as a tool to study conjectures related to cluster algebras ([16]). Another example is the study of self-injective algebras, that is, algebras $A$ such that $A \simeq D A$ as right $A$-modules. Indeed, to any algebra $A$ is associated the repetitive category $\widehat{A}$, which is a Galois covering with group $\mathbb{Z}$ of the trivial extension $A \ltimes D A$ (see [29]). Assume that some group $G$ acts freely on $\widehat{A}$ thus defining a Galois covering $\widehat{A} \rightarrow \widehat{A} / G$ with group $G$. If $\widehat{A} / G$ is a finite dimensional algebra, then it is self-injective. It is known that every self-injective algebra $\Lambda$ is of polynomial growth if and only if $\Lambda$ is a socle (geometric) deformation of an algebra of the form $\hat{A} / G$ where $A$ is a tilted algebra of Dynkin type or Euclidean type or a tubular algebra, and $G$ is an admissible infinite cyclic group of automorphisms of $\hat{A}([46$, 49]). Recently, the class of self-injective algebras of piecewise hereditary type has been the subject of many studies (see [15], 31], 37], [50, [51, [52]).

In this text we investigate the Galois coverings of piecewise hereditary algebras. The Galois coverings of algebras and, more generally, of $k$-categories, were introduced in [17, 41] for the classification of representation-finite algebras. Consider any finite dimensional algebra $A$ as a locally bounded $k$-category: If $1=$ $e_{1}+\cdots+e_{n}$ is a decomposition of the unity into primitive orthogonal idempotents, then $\mathrm{ob}(A)=\left\{e_{1}, \ldots, e_{n}\right\}$ and the space of morphisms from $e_{i}$ to $e_{j}$ is $e_{j} A e_{i}$. Then a Galois covering of the $k$-category $A$ is a $k$-linear functor $F: \mathcal{C} \rightarrow A$ where $\mathcal{C}$ is a $k$-category endowed with a free action of $G$, that is, $G$ acts freely on ob $(\mathcal{C})$, such that $F \circ g=F$ for every $g \in G$ and the induced quotient functor $\mathcal{C} / G \rightarrow A$ is an isomorphism $([17])$. In such a situation, $\bmod \mathcal{C}$ and $\bmod A$ are related by the so-called push-down functor $F_{\lambda}: \bmod \mathcal{C} \rightarrow \bmod A$, that is, the extension-of-scalars functor. Often, $F_{\lambda}$ allows nice comparisons between $\bmod \mathcal{C}$ and $\bmod A$. For example, the action of $G$ on $\mathcal{C}$ naturally defines an action $(g, X) \mapsto{ }^{g} X$ of $G$ on $\mathcal{C}$-modules. When this action is free on indecomposable $\mathcal{C}$-modules, $F_{\lambda}$ defines an isomorphism of translation quivers between $\Gamma(\bmod \mathcal{C}) / G$ and a union of some components of the Auslander-Reiten quiver $\Gamma(\bmod A)$ of $A($ see $[14$, [17]).

The comparisons allowed by the covering techniques naturally raise the following questions: Given an algebra $A$, is it possible to describe all the Galois coverings of $A$ (in particular, does $A$ admit a universal Galois covering, as happens in topology)? Is it possible to characterise the simple connectedness of $A$ ? We say that $A$ is simply connected if it admits no Galois covering with nontrivial group by a connected and locally bounded $k$-category. If $A$ is triangular, this is equivalent (see [47, 4.2]) to requiring that the fundamental groups of the presentations of $A$ by quiver and relations (in the sense of [38]) are all trivial. In view of the above discussion on self-injective algebras, these questions are relevant when $A$ is piecewise hereditary of type $Q$. In case $A=k Q$, the answers are well known: The Galois coverings of $k Q$ correspond to those of the underlying graph of $Q$; and $k Q$ is simply connected if and only if $Q$ is a tree, which is also equivalent to the vanishing of the first Hochschild cohomology group $\mathrm{HH}^{1}(k Q)$ ([11, [20]). Also, one can wonder if the data of the Galois coverings of $A$ and the simple connectedness of $A$ depend only on the bounded derived category $\mathcal{D}^{b}(\bmod A)$. Again, it is natural to treat this problem for piecewise hereditary algebras. Up to now, there are no general solutions to the above problems. The question of the description of the Galois coverings and that of the characterisation of simple connectedness have found a satisfactory answer in the case of standard representation-finite algebras $([9,47])$. This is mainly due to the fact that the Auslander-Reiten quiver is connected and completely describes 
the module category in this case. However, the infinite-representation case seems to be more complicated. As an example, there exist string algebras which admit no universal Galois covering ([34]).

In the present text, we study the above problems when $A$ is piecewise hereditary. Note that they have already been studied in that context. We say that $\mathcal{D}^{b}(\bmod A)$ is cycle-finite if every oriented cycle of nonzero nonisomorphisms between indecomposables in $\mathcal{D}^{b}(\bmod A)$ lies entirely in a tube. It was proved in 44, [5] that $\mathcal{D}^{b}(\bmod A)$ is cycle-finite if and only if $A$ is derived equivalent to a hereditary algebra of Dynkin or Euclidean type or to a tubular canonical algebra; moreover, such an algebra is simply connected if and only if it is not piecewise hereditary of type $\widetilde{\mathbb{A}}$ and, therefore, if and only if the first Hochschild cohomology group vanishes. Finally, the piecewise hereditary algebras of type $\widetilde{\mathbb{A}}$ have been described by quiver and relations in [3] so their Galois coverings are fairly well understood. In this text we prove the following main result.

Theorem A. Let $A$ be a connected algebra derived equivalent to a hereditary abelian category $\mathcal{H}$ whose oriented graph $\overrightarrow{\mathcal{K}}_{\mathcal{H}}$ of tilting objects is connected. Then $A$ admits a universal Galois covering $\widetilde{\mathcal{C}} \rightarrow A$ with group a free group $\pi_{1}(A)$ uniquely determined by $\mathcal{D}^{b}(\bmod A)$. This means that $\widetilde{\mathcal{C}}$ is connected and locally bounded and for any Galois covering $\mathcal{C} \rightarrow A$ with group $G$ where $\mathcal{C}$ is connected and locally bounded there exists a commutative diagram:

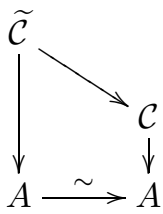

where the bottom horizontal arrow is an isomorphism extending the identity map on $\mathrm{ob}(A)$. Moreover, $\widetilde{\mathcal{C}} \rightarrow \mathcal{C}$ is Galois with group $N$ such that there is an exact sequence of groups $1 \rightarrow N \rightarrow \pi_{1}(A) \rightarrow G \rightarrow 1$.

Finally, if $A$ is piecewise hereditary of type $Q$, then $\pi_{1}(A)$ is the fundamental group $\pi_{1}(Q)$ of the underlying graph of $Q$ and, otherwise, the rank of $\pi_{1}(A)$ equals $\operatorname{dim}_{k} \mathrm{HH}^{1}(A)$ (which is 0 or 1 ).

We refer the reader to the next section for a reminder on $\overrightarrow{\mathcal{K}}_{\mathcal{H}}$. Recall that, by 27, the assumption on $A$ is satisfied if $A$ is piecewise hereditary of type $Q$ (in particular, if $A$ is tilted of type $Q$ ). However, given a hereditary abelian category $\mathcal{H}$ of canonical type, it is still an open problem to know whether $\overrightarrow{\mathcal{K}}_{\mathcal{H}}$ is connected or not (see [35. for a characterisation of hereditary abelian categories of canonical type). Barot, Kussin and Lenzing announced a solution to this problem in the tubular case (at the International Conference on Cluster Algebras and Related Topics, Mexico 2008), but the answer for hereditary abelian categories of wild canonical type is still unknown. Therefore, Theorem A generalises the description of the Galois coverings of piecewise hereditary algebras with cycle-finite derived categories to piecewise hereditary algebras of hereditary type (and possibly to piecewise hereditary algebras of wild canonical type).

Note that Theorem $\mathrm{A}$ implies that the Galois coverings of a piecewise hereditary algebra of type $Q$ are determined by the factor groups of $\pi_{1}(Q)$. Also, it shows that the data of the Galois coverings is an invariant of the derived category. Therefore, 
so does the simple connectedness. Using the fact that the Hochschild cohomology is invariant under derived equivalences ([40], see also [20, 30]), we deduce the following corollary of our main result.

Corollary B. Let $A$ be as in Theorem $\mathrm{A}$, The following are equivalent:

(a) $A$ is simply connected.

(b) $\mathrm{HH}^{1}(A)=0$.

If $A$ is piecewise hereditary of type $Q$, then (a) and (b) are also equivalent to:

(c) $Q$ is a tree.

This corollary generalises some of the results of [1, 5] which studied the same characterisation for tilted algebras of Euclidean type and for tame tilted algebras. Also, it gives a new class of algebras for which the following question of Skowroński ([48, $\mathrm{Pb}, 1])$ has a positive answer: Is A simply connected if and only if $\mathrm{HH}^{1}(A)=$ 0 ? Originally, this question was asked for tame triangular algebras. Note that this equivalence does not always hold true for wild algebras. Indeed, consider the wild algebra ([48, 3.4]) given by the quiver

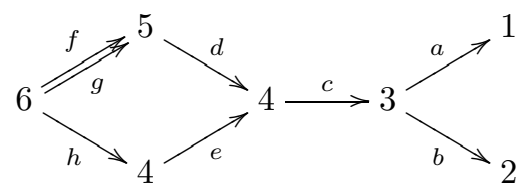

bound by the relations $f d c a-h e c a=0, g d c b-h e c b=0, g d c a=0$. Then $A$ is simply connected because it has the separation property, and $\operatorname{dim} \mathrm{HH}^{1}(A)=1$.

The methods we use to prove Theorem A allow us to prove the last main result of this text. It shows that the Galois coverings have a nice behaviour for piecewise hereditary algebras.

Theorem C. Let $A$ be piecewise hereditary of type $Q$ and let $F: \mathcal{C} \rightarrow A$ be a Galois covering with group $G$ where $\mathcal{C}$ is connected and locally bounded. Then $\mathcal{C}$ is piecewise hereditary of type $Q^{\prime}$, where $Q^{\prime}$ is a quiver such that there exists a Galois covering of quivers $Q^{\prime} \rightarrow Q$ with group $G$.

We now give some explanations on the proof of Theorem A For unexplained notions, we refer the reader to the next section. Assume that $A$ is piecewise hereditary. It is known from [24, Thm. 2.6] that there exists an algebra $B$ such that $A \simeq \operatorname{End}_{\mathcal{D}^{b}(\bmod B)}(X)$ for some tilting complex $X \in \mathcal{D}^{b}(\bmod B)$ and such that $B$ has one of the following forms:

(1) $B=k Q$, with $Q$ a finite quiver with no oriented cycles.

(2) $B$ is a squid algebra.

It is easy to check that Theorem $\mathrm{A}$ holds true for path algebras of quivers and for squid algebras. Therefore, we are reduced to proving that Theorem $\mathrm{A}$ holds true for $A$ and only if it holds true for $\operatorname{End}_{\mathcal{D}^{b}(\bmod A)}(T)$ for any tilting complex $T \in \mathcal{D}^{b}(\bmod A)$. Roughly speaking, we need a correspondence between the Galois coverings of $A$ and those of $\operatorname{End}_{\mathcal{D}^{b}(\bmod A)}(T)$. Therefore, we use a construction introduced in [33] for tilting modules: Given a Galois covering $F: \mathcal{C} \rightarrow A$ with group $G$, the push-down functor $F_{\lambda}: \bmod \mathcal{C} \rightarrow \bmod A$ is exact and therefore induces an exact functor $F_{\lambda}: \mathcal{D}^{b}(\bmod \mathcal{C}) \rightarrow \mathcal{D}^{b}(\bmod A)$. Also, the $G$-action on modules extends to a $G$-action on $\mathcal{D}^{b}(\bmod \mathcal{C})$ by triangle automorphisms. Now, let $T \in \mathcal{D}^{b}(\bmod A)$ 
be a tilting complex and let $T=T_{1} \oplus \cdots \oplus T_{n}$ be an indecomposable decomposition. Assume that the following conditions hold true for every $i \in\{1, \ldots, n\}$ :

$\left(H_{1}\right)$ There exists an indecomposable $\mathcal{C}$-module $\widetilde{T}_{i}$ such that $F_{\lambda} \widetilde{T}_{i}=T_{i}$.

$\left(H_{2}\right)$ The stabiliser $\left\{g \in G \mid{ }^{g} \widetilde{T}_{i} \simeq \widetilde{T}_{i}\right\}$ is the trivial group.

Under these assumptions, the complexes ${ }^{g} \widetilde{T}_{i}$ (for $g \in G$ and $i \in\{1, \ldots, n\}$ ) form a full subcategory of $\mathcal{D}^{b}(\bmod \mathcal{C})$ which we denote by $\operatorname{End}_{\mathcal{D}^{b}(\bmod \mathcal{C})}(\widetilde{T})$. Then $F_{\lambda}: \mathcal{D}^{b}(\bmod \mathcal{C}) \rightarrow \mathcal{D}^{b}(\bmod A)$ induces a Galois covering with group $G$ :

$$
\begin{aligned}
\operatorname{End}_{\mathcal{D}^{b}(\bmod \mathcal{C})}(\widetilde{T}), & \rightarrow \operatorname{End}_{\mathcal{D}^{b}(\bmod A)}(T), \\
{ }^{g} \widetilde{T}_{i} & \mapsto T_{i}, \\
{ }^{g} \widetilde{T}_{i} \stackrel{u}{\rightarrow}{ }^{h} \widetilde{T}_{j} & \mapsto T_{i} \stackrel{F_{\lambda}(u)}{\longrightarrow} T_{j} .
\end{aligned}
$$

Hence $\left(H_{1}\right)$ and $\left(H_{2}\right)$ are technical conditions which allow one to associate a Galois covering of $\operatorname{End}_{\mathcal{D}^{b}(\bmod A)}(T)$ to a Galois covering of $A$. In particular, if $A$ admits a universal Galois covering, then the associated Galois covering of $\operatorname{End}_{\mathcal{D}^{b}(\bmod A)}(T)$ is a good candidate for being a universal Galois covering. This is indeed the case provided that the following technical condition is satisfied:

$\left(H_{3}\right)$ If $\psi: A \stackrel{\sim}{\rightarrow} A$ is an automorphism such that $\psi(x)=x$ for every $x \in \mathrm{ob}(A)$, then $\psi_{\lambda} T_{i} \simeq T_{i}$, for every $i$.

We therefore need to prove the assertions $\left(H_{1}\right),\left(H_{2}\right)$ and $\left(H_{3}\right)$ for every Galois covering $F: \mathcal{C} \rightarrow A$ and every tilting complex $T \in \mathcal{D}^{b}(\bmod A)$.

The text is therefore organised as follows. In Section 1, we recall some useful definitions and fix some notation. In Section 2, we define the exact functor $F_{\lambda}: \mathcal{D}^{b}(\bmod \mathcal{C}) \rightarrow \mathcal{D}^{b}(\bmod A)$ associated to a Galois covering $F: \mathcal{C} \rightarrow A$. In Section 3, we introduce elementary transformations on tilting complexes using approximations. The main result of the section asserts that for $A$ piecewise hereditary of type $Q$ and for any tilting complexes $T, T^{\prime}$, there exists a sequence of elementary transformations relating $T$ and $T^{\prime}$. We prove the assertions $\left(H_{1}\right),\left(H_{2}\right)$ and $\left(H_{3}\right)$ in Section 4 using the elementary transformations. We prove Theorem $\mathrm{C}$ as an application of these results. Then, in Section 5 we establish a correspondence between the Galois coverings of $A$ and those of $\operatorname{End}_{\mathcal{D}^{b}(\bmod A)}(T)$ for every tilting complex $T$. Finally, we prove Theorem $\mathrm{A}$ and Corollary B in Section 6 .

\section{Definitions and notation}

Modules over $k$-categories. We refer the reader to [7] for the definition of $k$ categories and locally bounded $k$-categories. All locally bounded $k$-categories are assumed to be small and all functors between $k$-categories are assumed to be $k$-linear (our module categories and derived categories will be skeletally small). Let $\mathcal{C}$ be a $k$-category. Following [7], a (right) $\mathcal{C}$-module is a $k$-linear functor $M: \mathcal{C}^{o p} \rightarrow \mathrm{MOD} k$ where MOD $k$ is the category of $k$-vector spaces. The category of $\mathcal{C}$-modules is denoted by $\operatorname{MOD} \mathcal{C}$. A module $M \in \operatorname{MOD} \mathcal{C}$ is called finite dimensional if $\sum_{x \in \operatorname{ob}(\mathcal{C})} \operatorname{dim}_{k} M(x)<\infty$. The category of finite dimensional $\mathcal{C}$-modules is denoted by $\bmod \mathcal{C}$. Note that the indecomposable projective $\mathcal{C}$-module associated to $x \in \mathrm{ob}(\mathcal{C})$ is the representable functor $\mathcal{C}(-, x)$. The projective dimension of a $\mathcal{C}$-module $X$ is denoted by $\operatorname{pd}_{\mathcal{C}}(X)$. If $X \in \bmod \mathcal{C}$, then $\operatorname{add}(X)$ denotes the smallest full subcategory of $\bmod \mathcal{C}$ containing $X$ and closed under direct summands and direct sums. We refer the reader to 2 for notions on tilting theory. If $A$ is an algebra, 
an $A$-module $T$ is called tilting if: (a) $T$ is multiplicity-free; (b) $\operatorname{pd}_{A}(T) \leqslant 1$; (c) $\operatorname{Ext}_{A}^{1}(T, T)=0$; (d) for every indecomposable projective $A$-module $P$ there is an exact sequence $0 \rightarrow P \rightarrow X \rightarrow Y \rightarrow 0$ in $\bmod A$ where $X, Y \in \operatorname{add}(T)$. Let $\mathcal{H}$ be a hereditary abelian category. An object $T \in \mathcal{H}$ is called tilting (see [24]) if: (a) $T$ is multiplicity-free; (b) $\operatorname{Ext}_{\mathcal{H}}^{1}(T, T)=0 ;(\mathrm{c})$ whenever $\operatorname{Hom}_{\mathcal{H}}(T, X)=\operatorname{Ext}_{\mathcal{H}}^{1}(T, X)=0$ for $X \in \mathcal{H}$, then $X=0$. The set of isomorphism classes of tilting objects in $\mathcal{H}$ has a partial order such that $T \leqslant T^{\prime}$ if and only if Fac $T \subseteq \operatorname{Fac} T^{\prime}$ where Fac $T$ is the class of epimorphic images of direct sums of copies of $T$. The Hasse diagram of this poset is denoted by $\overrightarrow{\mathcal{K}}_{\mathcal{H}}$ and called the oriented graph of tilting objects in $\mathcal{H}$ (see 27] for more details). A complex $T$ in $\mathcal{D}^{b}(\mathcal{H})\left(\operatorname{or} \mathcal{D}^{b}(\bmod A)\right)$ is called tilting if: (a) $T$ is multiplicity-free; (b) $\operatorname{Hom}(T, T[i])=0$ for all $i \neq 0$; (c) $T$ generates $\mathcal{D}^{b}(\mathcal{H})$ (or $\mathcal{D}^{b}(\bmod A)$, respectively) as a triangulated category.

If $\mathcal{A}$ is an additive category, then ind $\mathcal{A}$ denotes the full subcategory of all indecomposable objects of $\mathcal{A}$.

Galois coverings of $k$-categories. Let $F: \mathcal{E} \rightarrow \mathcal{B}$ be a Galois covering with group $G$ between $k$-categories (see the introduction). It is called connected if both $\mathcal{C}$ and $\mathcal{B}$ are connected and locally bounded. Let $A$ be a connected and locally bounded $k$-category and $x_{0} \in \mathrm{ob}(A)$. A pointed Galois covering $F:(\mathcal{C}, x) \rightarrow\left(A, x_{0}\right)$ is a connected Galois covering $F: \mathcal{C} \rightarrow A$ endowed with $x \in \mathrm{ob}(\mathcal{C})$ such that $F(x)=x_{0}$. A morphism of pointed Galois coverings $F \stackrel{u}{\rightarrow} F^{\prime}$ from $F:(\mathcal{C}, x) \rightarrow\left(A, x_{0}\right)$ to $F^{\prime}:\left(\mathcal{C}^{\prime}, x^{\prime}\right) \rightarrow\left(A, x_{0}\right)$ is a functor $u: \mathcal{C} \rightarrow \mathcal{C}^{\prime}$ such that $F^{\prime} \circ u=F$ and $u(x)=x^{\prime}$. Note that, given $F$ and $F^{\prime}$, there is at most one such morphism ([32, Lem. 3.1]). This defines the category $\operatorname{Gal}\left(A, x_{0}\right)$ of pointed Galois coverings. If $F \in \operatorname{Gal}\left(A, x_{0}\right)$, then we let $F \rightarrow$ be the full subcategory of $\operatorname{Gal}\left(A, x_{0}\right)$ whose objects are the pointed Galois coverings $F^{\prime} \in \operatorname{Gal}\left(A, x_{0}\right)$ such that there exists a morphism $F \rightarrow F^{\prime}$.

Covering properties on module categories (7, 41]). Let $F: \mathcal{E} \rightarrow \mathcal{B}$ be a Galois covering with group $G$. The $G$-action on $\mathcal{E}$ defines a $G$-action on MOD $\mathcal{E}$ : If $M \in \operatorname{MOD} \mathcal{E}$ and $g \in G$, then ${ }^{g} M:=F \circ g^{-1} \in \operatorname{MOD} \mathcal{E}$. If $X \in \operatorname{MOD} \mathcal{E}$, the stabiliser of $X$ is the subgroup $G_{X}:=\left\{g \in G \mid{ }^{g} X \simeq X\right\}$ of $G$. The Galois covering $F$ defines two exact functors: The extension-of-scalars functor $F_{\lambda}:$ MOD $\mathcal{E} \rightarrow$ MOD $\mathcal{B}$ which is called the push-down functor and the restriction-of-scalars functor $F$.: MOD $\mathcal{B} \rightarrow$ MOD $\mathcal{E}$ which is called the pull-up functor. They form an adjoint pair $\left(F_{\lambda}, F_{\text {. }}\right)$ and $F_{\lambda}$ is $G$-invariant, that is, $F_{\lambda} \circ g=F_{\lambda}$ for every $g \in G$. We refer the reader to [7] for details on $F_{\lambda}$ and $F_{\text {. For }}$. Fony $M, N \in \bmod \mathcal{E}$, the following maps induced by $F_{\lambda}$ are bijective:

$$
\bigoplus_{g \in G} \operatorname{Hom}_{\mathcal{E}}\left({ }^{g} M, N\right) \rightarrow \operatorname{Hom}_{\mathcal{B}}\left(F_{\lambda} M, F_{\lambda} N\right)
$$

and

$$
\bigoplus_{g \in G} \operatorname{Hom}_{\mathcal{E}}\left(M,{ }^{g} N\right) \rightarrow \operatorname{Hom}_{\mathcal{B}}\left(F_{\lambda} M, F_{\lambda} N\right) .
$$

An indecomposable module $X \in \bmod \mathcal{B}$ is called of the first kind with respect to $F$ if and only if $F_{\lambda} \widetilde{X} \simeq X$ for some $\widetilde{X} \in \bmod \mathcal{E}$ (necessarily indecomposable). In such a case, one may choose $\widetilde{X}$ such that $F_{\lambda} \tilde{X}=X$. More generally, $X \in \bmod \mathcal{B}$ is called of the first kind with respect to $F$ if and only if it is the direct sum of indecomposable $\mathcal{B}$-modules of the first kind with respect to $F$. 


\section{Covering techniques on the Bounded Derived CATEgory}

Let $F: \mathcal{C} \rightarrow A$ be a Galois covering with group $G$ and, with $\mathcal{C}$ and $A$, locally bounded categories of finite global dimension. The $G$-action on $\bmod \mathcal{C}$ naturally defines a $G$-action on $\mathcal{D}^{b}(\bmod \mathcal{C})$, still denoted by $(g, M) \mapsto{ }^{g} M$, by triangle automorphisms. We introduce an exact functor $F_{\lambda}: \mathcal{D}^{b}(\bmod \mathcal{C}) \rightarrow \mathcal{D}^{b}(\bmod A)$ induced by $F_{\lambda}: \bmod \mathcal{C} \rightarrow \bmod A$.

Proposition 2.1. There exists an exact functor $F_{\lambda}: \mathcal{D}^{b}(\bmod \mathcal{C}) \rightarrow \mathcal{D}^{b}(\bmod A)$ such that the following diagram commutes:

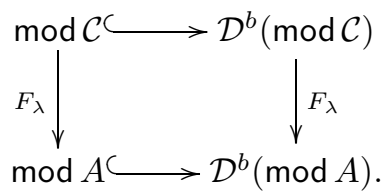

The functor $F_{\lambda}: \mathcal{D}^{b}(\bmod \mathcal{C}) \rightarrow \mathcal{D}^{b}(\bmod A)$ has the covering property, that is, it is $G$-invariant and the two following maps are linear bijections for every $M, N \in$ $\mathcal{D}^{b}(\bmod \mathcal{C})$ :

$$
\begin{aligned}
& \bigoplus_{g \in G} \mathcal{D}^{b}(\bmod \mathcal{C})\left({ }^{g} M, N\right) \stackrel{F_{\lambda}}{\longrightarrow} \mathcal{D}^{b}(\bmod A)\left(F_{\lambda} M, F_{\lambda} N\right) \\
& \bigoplus_{g \in G} \mathcal{D}^{b}(\bmod \mathcal{C})\left(M,{ }^{g} N\right) \stackrel{F_{\lambda}}{\longrightarrow} \mathcal{D}^{b}(\bmod A)\left(F_{\lambda} M, F_{\lambda} N\right)
\end{aligned}
$$

Proof. The existence and exactness of $F_{\lambda}: \mathcal{D}^{b}(\bmod \mathcal{C}) \rightarrow \mathcal{D}^{b}(\bmod A)$ follow from the exactness of $F_{\lambda}: \bmod \mathcal{C} \rightarrow \bmod A$. On the other hand, $F_{\lambda}$ induces an additive functor $F_{\lambda}: \mathcal{K}^{b}(\bmod \mathcal{C}) \rightarrow \mathcal{K}^{b}(\bmod A)$ between bounded homotopy categories of complexes. It easily checked that it has the covering property in the sense of the proposition. Since $A$ and $\mathcal{C}$ have finite global dimension, we deduce that $F_{\lambda}: \mathcal{D}^{b}(\bmod \mathcal{C}) \rightarrow \mathcal{D}^{b}(\bmod A)$ has the covering property.

Remark 2.2. It follows from the preceding proposition that $F_{\lambda}: \mathcal{D}^{b}(\bmod \mathcal{C}) \rightarrow$ $\mathcal{D}^{b}(\bmod A)$ is faithful.

We are mainly interested in indecomposable objects $X \in \mathcal{D}^{b}(\bmod A)$ which are of the form $F_{\lambda} \tilde{X}$ for some $\tilde{X} \in \mathcal{D}^{b}(\bmod \mathcal{C})$. The following shows that the possible objects $\widetilde{X}$ lie in the same $G$-orbit for a given $X$.

Lemma 2.3. Let $X, Y \in \mathcal{D}^{b}(\bmod \mathcal{C})$ be such that $F_{\lambda} X$ and $F_{\lambda} Y$ are indecomposable and isomorphic in $\mathcal{D}^{b}(\bmod A)$. Then $X \simeq{ }^{g} Y$ for some $g \in G$.

Proof. Let $u: F_{\lambda} X \rightarrow F_{\lambda} Y$ be an isomorphism in $\mathcal{D}^{b}(\bmod A)$. By 2.1, there exists $\left(u_{g}\right)_{g} \in \bigoplus_{g \in G} \mathcal{D}^{b}(\bmod \mathcal{C})\left(X,{ }^{g} Y\right)$ such that $u=\sum_{g \in G} F_{\lambda}\left(u_{g}\right)$. Since $F_{\lambda} X$ and $F_{\lambda} Y$ are indecomposable, there exists $g_{0} \in G$ such that $F_{\lambda}\left(u_{g_{0}}\right): F_{\lambda} X \rightarrow F_{\lambda} Y$ is an isomorphism. Since $F_{\lambda}: \mathcal{D}^{b}(\bmod \mathcal{C}) \rightarrow \mathcal{D}^{b}(\bmod A)$ is exact and faithful, $u_{g_{0}}: X \rightarrow$ ${ }^{g_{0}} Y$ is an isomorphism in $\mathcal{D}^{b}(\bmod \mathcal{C})$.

\section{TRANSFORMing TILTING COMPLEXES INTO TILTING MODUlES}

Let $\mathcal{H}$ be a hereditary abelian category over $k$ with finite dimensional Homspaces, split idempotents and tilting objects. Let $n$ be the rank of its Grothendieck 
group. For short, we set $\operatorname{Hom}:=\operatorname{Hom}_{\mathcal{D}^{b}(\mathcal{H})}$ and $\operatorname{Ext}^{i}(X, Y):=\operatorname{Hom}_{\mathcal{D}^{b}(\mathcal{H})}(X, Y[i])$. We write $\mathcal{T}$ for the class of objects $T \in \mathcal{D}^{b}(\mathcal{H})$ such that:

(a) $T$ is multiplicity-free and has $n$ indecomposable summands.

(b) $\operatorname{Ext}^{i}(T, T)=0$ for every $i \geqslant 1$.

We identify an object in $\mathcal{T}$ with its isomorphism class. A complex $T$ lies in $\mathcal{T}$ if and only if $T[1] \in \mathcal{T}$. Also, all tilting complexes in $\mathcal{D}^{b}(\mathcal{H})$ and, therefore, all tilting objects in $\mathcal{H}$, lie on $\mathcal{T}$. Given $T \in \mathcal{D}^{b}(\mathcal{H})$, we let $\langle T\rangle$ be the smallest full subcategory of $\mathcal{D}^{b}(\mathcal{H})$ containing $T$ and stable under direct sums, direct summands and shifts in either direction. The aim of this section is to define elementary transformations on objects in $\mathcal{T}$ which, by repetition, allow one to relate any two objects in $\mathcal{T}$ when $\overrightarrow{\mathcal{K}}_{\mathcal{H}}$ is connected. For this purpose, we introduce some notation. Given $T \in \mathcal{T}$, we have a unique decomposition $T=Z_{0}\left[i_{0}\right] \oplus Z_{1}\left[i_{0}+1\right] \oplus \cdots \oplus Z_{l}\left[i_{0}+l\right]$ where $Z_{0}, \ldots, Z_{l} \in \mathcal{H}$ and $Z_{0}, Z_{l} \neq 0$. Here, $Z_{i}$ need not be indecomposable. We let $r(T)$ be the number of indecomposable summands of $Z_{1}\left[i_{0}+1\right] \oplus \cdots \oplus Z_{l}\left[i_{0}+l\right]$. Note that: $r(T) \in\{0, \ldots, n-1\} ; r(T)=0$ if and only if $T\left[-i_{0}\right]$ is a tilting object in $\mathcal{H}$; and $r(T)=r(T[1])$. We are interested in transformations which map an object $T \in \mathcal{T}$ to $T^{\prime}$ such that $r\left(T^{\prime}\right)<r(T)$. Hence, by repeating the process, we may end up with a tilting object in $\mathcal{H}$ (up to a shift).

Transformations of the first kind. Our first elementary transformation is given by the following lemma.

Lemma 3.1. Let $T \in \mathcal{T}$. There exists $T^{\prime} \in \mathcal{T}$ such that $T^{\prime} \in\langle T\rangle, r\left(T^{\prime}\right) \leqslant r(T)$ and $T^{\prime}=Z_{0}^{\prime} \oplus Z_{1}^{\prime}[1] \oplus \cdots \oplus Z_{l^{\prime}}^{\prime}\left[l^{\prime}\right]$ where:

(a) $Z_{0}^{\prime}, \ldots, Z_{l^{\prime}}^{\prime} \in \mathcal{H}$ and $Z_{0}^{\prime}, Z_{l^{\prime}}^{\prime} \neq 0$.

(b) $\operatorname{Hom}\left(Z_{0}^{\prime}, Z_{1}^{\prime}[1]\right) \neq 0$ if $l^{\prime} \neq 0$.

Proof. Given $T^{\prime} \in\langle T\rangle$ we have the unique decomposition $T^{\prime}=Z_{0}^{\prime}\left[i_{0}^{\prime}\right] \oplus Z_{1}^{\prime}\left[i_{0}^{\prime}+\right.$ 1] $\oplus \cdots \oplus Z_{l}^{\prime}\left[i_{0}^{\prime}+l^{\prime}\right]$ as explained at the beginning of the section. We choose $T^{\prime} \in$ $\langle T\rangle \cap \mathcal{T}$ such that $r\left(T^{\prime}\right) \leqslant r(T)$ and such that the pair $\left(l^{\prime}, r\left(T^{\prime}\right)\right)$ is minimal for the lexicographical order. We may assume that $i_{0}^{\prime}=0$. We prove that $T^{\prime}$ satisfies (a) and (b). If $l^{\prime}=0$, there is nothing to prove. So we assume that $l^{\prime}>0$. Assume first that $Z_{1}^{\prime}=0$. Then we let $T^{\prime \prime}$ be as follows:

$$
T^{\prime \prime}:=Z_{0}^{\prime} \oplus Z_{2}^{\prime}[1] \oplus Z_{3}^{\prime}[2] \oplus \cdots \oplus Z_{l^{\prime}}^{\prime}\left[l^{\prime}-1\right] .
$$

Hence $T^{\prime \prime} \in\left\langle T^{\prime}\right\rangle=\langle T\rangle$. Also, Ext ${ }^{i}\left(T^{\prime \prime}, T^{\prime \prime}\right)=0$ for every $i \geqslant 1$ because $T^{\prime} \in \mathcal{T}$ and $\mathcal{H}$ is hereditary. Finally, $T^{\prime \prime}$ is the direct sum of $n$ pairwise nonisomorphic indecomposable objects. Thus, $T^{\prime \prime} \in\langle T\rangle \cap \mathcal{T}$ and $\left(l^{\prime}-1, r\left(T^{\prime \prime}\right)\right)<\left(l^{\prime}, r\left(T^{\prime}\right)\right)$ which contradicts the minimality of $\left(l^{\prime}, r\left(T^{\prime}\right)\right)$. So $Z_{1}^{\prime} \neq 0$. Now, assume that $\operatorname{Hom}\left(Z_{0}^{\prime}, Z_{1}^{\prime}[1]\right)=0$. We let $T^{\prime \prime}$ be the following object:

$$
T^{\prime \prime}:=\left(Z_{0}^{\prime} \oplus Z_{1}^{\prime}\right) \oplus Z_{2}^{\prime}[2] \oplus Z_{3}^{\prime}[3] \oplus \cdots \oplus Z_{l^{\prime}}^{\prime}\left[l^{\prime}\right] .
$$

As above, we have $T^{\prime \prime} \in\langle T\rangle \cap \mathcal{T}$ and $\left(l^{\prime}, r\left(T^{\prime \prime}\right)\right)<\left(l^{\prime}, r\left(T^{\prime}\right)\right)$ which contradicts the minimality of $\left(l^{\prime}, r\left(T^{\prime}\right)\right)$. So $\operatorname{Hom}\left(Z_{0}^{\prime}, Z_{1}^{\prime}[1]\right) \neq 0$.

If $T, T^{\prime} \in \mathcal{T}$ are as in the preceding lemma we say that $T$ and $T^{\prime}$ are related by a transformation of the first kind. 
Transformations of the second kind. We now turn to the second elementary transformation. It is inspired by the characterisation of the quiver of tilting objects in hereditary categories (see [27] and also [8] for the corresponding construction in cluster categories). Let $T, T^{\prime} \in \mathcal{T}$ be such that $T=X \oplus \bar{T}$ with $X$ indecomposable, $T^{\prime}=Y \oplus \bar{T}$ with $Y$ indecomposable and there exists a triangle $X \stackrel{u}{\rightarrow} M \stackrel{v}{\rightarrow} Y \rightarrow$ $X[1]$ such that $u$ is a left minimal add $(\bar{T})$-approximation or $v$ is a right minimal $\operatorname{add}(\bar{T})$-approximation. In such a situation, we say that $T^{\prime}$ is obtained from $T$ by a transformation of the second kind.

Remark 3.2. (a) Both transformations (of the first or of the second kind) make sense in $\mathcal{D}^{b}(\bmod A)$ for any algebra $A$ of finite global dimension.

(b) Following [27, if $T \rightarrow T^{\prime}$ is an arrow in $\overrightarrow{\mathcal{K}}_{\mathcal{H}}$, then $T$ and $T^{\prime}$ are related by a transformation of the second kind.

Note that, with the previous notation, both $u$ and $v$ are minimal $\operatorname{add}(\bar{T})$ approximations as shown in the following lemma.

Lemma 3.3. Let $T \in \mathcal{T}$. Assume that $T=X \oplus \bar{T}$ with $X$ indecomposable.

(a) Let $X \stackrel{u}{\rightarrow} M \stackrel{v}{\rightarrow} Y \rightarrow X[1]$ be a triangle where $u$ is a left minimal $\operatorname{add}(\bar{T})$ approximation. Then $v$ is a right minimal $\operatorname{add}(\bar{T})$-approximation.

(b) Let $Y \stackrel{u}{\rightarrow} M \stackrel{v}{\rightarrow} X \rightarrow Y$ [1] be a triangle where $v$ is a right minimal add $(\bar{T})$ approximation. Then $u$ is a left minimal $\operatorname{add}(\bar{T})$-approximation.

Proof. We only prove (a) because the proof of (b) is similar. Every morphism $\bar{T} \rightarrow Y$ factors through $v$ because $\operatorname{Hom}(\bar{T}, X[1])=0$. So $v$ is a right $\operatorname{add}(\bar{T})$ approximation. Let $\alpha: M \rightarrow M$ be a morphism such that $v \alpha=v$. So there exists $\lambda: M \rightarrow X$ such that $u \lambda=\alpha-\operatorname{ld}_{M}$. Note that $u$ is not a section because $T$ is multiplicity-free. So $u \lambda$ is nilpotent and $\alpha=\operatorname{ld}_{M}+u \lambda$ is an isomorphism. Therefore, $v$ is right minimal.

It is not true that any two objects $T, T^{\prime} \in \mathcal{T}$ can be related by a sequence of transformations of the second kind (whereas this is the case, for example, for tilting objects in a cluster category, $[8,3.5])$. However, we have the following result from 27.

Proposition 3.4. Assume that at least one of the two following assertions is true:

(a) $\mathcal{H}=\bmod k Q$ where $Q$ is a finite connected quiver without oriented cycles and of Dynkin type.

(b) $\mathcal{H}$ has no nonzero projective object and $\mathcal{D}^{b}(\mathcal{H})$ is triangle equivalent to $\mathcal{D}^{b}(\bmod k Q)$ with $Q$ a connected finite quiver without oriented cycles.

Then $\overrightarrow{\mathcal{K}}_{\mathcal{H}}$ is connected. In particular (see 3.2) for every tilting object $T, T^{\prime} \in \mathcal{H}$ there exists a sequence $T=T_{0}, \ldots, T_{l}=T^{\prime}$ of tilting objects in $\mathcal{H}$ such that $T_{i}$ and $T_{i+1}$ are related by a transformation of the second kind for every $i$.

We are going to prove that any $T \in \mathcal{T}$ can be related to some tilting object in $\mathcal{H}$ by a sequence of transformations of the first or of the second kind. Let $T \in \mathcal{T}$. With the notation established at the beginning of the section, assume that $r(T) \neq 0$ and $\operatorname{Hom}\left(Z_{0}, Z_{1}[1]\right) \neq 0$. Since the ordinary quiver of $\operatorname{End}(T)$ has no oriented cycles, 
there exists $M \in \operatorname{add}\left(Z_{1}\left[i_{0}+1\right]\right)$ indecomposable such that:

(1) $\operatorname{Hom}\left(Z_{0}\left[i_{0}\right], M\right) \neq 0$.

(2) $\operatorname{Hom}(Z, M)=0$ for any indecomposable direct summand $Z$ of $\bigoplus_{t=1}^{l} Z_{t}\left[i_{0}+\right.$ $t]$ not isomorphic to $M$.

Let $\bar{T}$ be such that $T=\bar{T} \oplus M$. Let $B \rightarrow M$ be a right minimal add $(\bar{T})$ approximation of $M$. Complete it into a triangle in $\mathcal{D}^{b}(\mathcal{H})$ :

$$
M^{*} \rightarrow B \rightarrow M \rightarrow M^{*}[1] .
$$

Lemma 3.5. With the above setting, let $T^{\prime}=\bar{T} \oplus M^{*}$. Then $T^{\prime} \in \mathcal{T}$ and $T, T^{\prime}$ are related by a transformation of the second kind. Moreover, $r\left(T^{\prime}\right)<r(T)$.

Proof. We only need to prove that $T^{\prime} \in \mathcal{T}$ and $r\left(T^{\prime}\right)<r(T)$. We may assume that $i_{0}=0$. By hypothesis on $M$, we have $B \in \operatorname{add}\left(Z_{0}\right) \subseteq \mathcal{H}$. Since $M \in \mathcal{H}[1]$, the triangle $\Delta$ does not split. We now list some properties on $T^{\prime}$. In most cases, the proof is due to arguments taken from [8, §6]. Although these arguments were originally given in the setting of cluster categories (that is, triangulated categories which are Calabi-Yau of dimension 2), it is easily verified that they still work in our situation (that is, the Calabi-Yau property is unnecessary):

(1) $\operatorname{Ext}^{1}\left(\bar{T}, M^{*}\right)=0($ (8, Lem. 6.3]).

(2) $\operatorname{Ext}^{i}\left(\bar{T}, M^{*}\right)=0$ for every $i \geqslant 2$. Indeed, applying $\operatorname{Hom}(\bar{T},-)$ to $\Delta$ gives the exact sequence

$$
0=\operatorname{Ext}^{i-1}(\bar{T}, M) \rightarrow \operatorname{Ext}^{i}\left(\bar{T}, M^{*}\right) \rightarrow \operatorname{Ext}^{i}(\bar{T}, B)=0 .
$$

(3) $\operatorname{Ext}^{i}\left(M^{*}, \bar{T}\right)=0$ for every $i \geqslant 1$. Indeed, applying $\operatorname{Hom}(-, \bar{T})$ to $\Delta$ gives the exact sequence

$$
0=\operatorname{Ext}^{i}(B, \bar{T}) \rightarrow \operatorname{Ext}^{i}\left(M^{*}, \bar{T}\right) \rightarrow \operatorname{Ext}^{i+1}(M, \bar{T})=0 .
$$

(4) The map $M^{*} \rightarrow B$ is a left minimal add $(\bar{T})$-approximation ([8, Lem. 6.4]).

(5) $M^{*}$ is indecomposable and does not lie on add $(\bar{T})([8$, Lems. 6.5, 6.6]). Therefore, $T^{\prime}$ is the direct sum of $n$ pairwise indecomposable objects.

(6) $M^{*} \in \mathcal{H}$. Indeed, $M$ is indecomposable and there are two nonzero maps $M[-1] \rightarrow M^{*}$ and $M^{*} \rightarrow B$ with $M[-1], B \in \mathcal{H}$.

(7) $\operatorname{Ext}^{1}\left(M^{*}, M^{*}\right)=0$ ([8, Lem. 6.7]).

(8) $\operatorname{Ext}^{i}\left(M^{*}, M^{*}\right)=0$ for every $i \geqslant 2$ because $M^{*}$ is indecomposable and $\mathcal{H}$ is hereditary.

The facts $(1)-(8)$ express that $T^{\prime} \in \mathcal{T}^{\prime}$. Moreover, $r\left(T^{\prime}\right)<r(T)$ because $M^{*} \in \mathcal{H}$ and $M \in \mathcal{H}[1]$.

Lemma 3.6. Let $T \in \mathcal{T}$. Let $\mathcal{A}$ be the smallest subclass of $\mathcal{T}$ containing $T$ and stable under transformations of the first or of the second kind. Then $\mathcal{A}$ contains a tilting object in $\mathcal{H}$.

Proof. Let $T^{\prime} \in \mathcal{A}$ be such that $r\left(T^{\prime}\right)$ is minimal for this property. Assume that $r\left(T^{\prime}\right)>0$. By 3.1 and 3.5, there exists $T^{\prime \prime} \in \mathcal{A}$ such that $r\left(T^{\prime \prime}\right)<r\left(T^{\prime}\right)$. This contradicts the minimality of $r\left(T^{\prime}\right)$. Hence, $r\left(T^{\prime}\right)=0$ and there exists an integer $i_{0}$ such that $T^{\prime}\left[-i_{0}\right]$ is a tilting object in $\mathcal{H}$ and lies in $\mathcal{A}$. 
The following result is a direct consequence of 3.4 and 3.6 .

Proposition 3.7. Assume that $\overrightarrow{\mathcal{K}}_{\mathcal{H}}$ is connected. Let $T \in \mathcal{T}$. Then $\mathcal{T}$ is the smallest subset of $\mathcal{T}$ containing $T$ and stable under transformations of the first or the second kind.

Remark 3.8. (a) A tilting object in $\mathcal{H}$ generates $\mathcal{D}^{b}(\mathcal{H})$. By definition of the two kinds of transformations, 3.7 implies, under the same hypotheses, that any $T \in \mathcal{T}$ generates $\mathcal{D}^{b}(\mathcal{H})$.

(b) Let $A$ be an algebra derived equivalent to a hereditary algebra. Then 3.4 implies that the conclusion of 3.7 holds true if one replaces $\mathcal{H}$ by $\bmod A$.

\section{Tilting COMPleXes OF THE FiRST KIND}

Throughout this section, we assume that $A$ is an algebra derived equivalent to a hereditary abelian category $\mathcal{H}$ such that $\overrightarrow{\mathcal{K}}_{\mathcal{H}}$ is connected. We denote by $n$ the rank of its Grothendieck group and fix $\Theta: \mathcal{D}^{b}(\mathcal{H}) \rightarrow \mathcal{D}^{b}(\bmod A)$, a triangle equivalence. We fix a Galois covering $F: \mathcal{C} \rightarrow A$ with group $G$ and with $\mathcal{C}$ locally bounded. We use 2.1 without reference. The aim of this section is to prove that the following facts hold true for any tilting complex $T \in \mathcal{D}^{b}(\bmod A)$ :

$\left(H_{1}\right)$ For every indecomposable direct summand $X$ of $T$, there exists $\tilde{X} \in$ $\mathcal{D}^{b}(\bmod \mathcal{C})$ such that $F_{\lambda} \tilde{X} \simeq X$ in $\mathcal{D}^{b}(\bmod A)$.

$\left(H_{2}\right) \quad \widetilde{X} \not{ }^{g} \widetilde{X}$ for every indecomposable direct summand $X$ of $T$ and $g \in G \backslash\{1\}$.

$\left(H_{3}\right)$ If $\psi: A \stackrel{\sim}{\longrightarrow} A$ is an automorphism such that $\psi(x)=x$ for every $x \in \mathrm{ob}(A)$, then $\psi_{\lambda} X \simeq X$ in $\mathcal{D}^{b}(\bmod A)$ for every indecomposable direct summand $X$ of $T$.

Some results presented in this section have been proved in [33, §3] in the case of tilting modules.

Proof of assertion $\left(H_{1}\right)$. In this paragraph, we prove the following.

Proposition 4.1. Let $A$ be as above. Let $T \in \mathcal{D}^{b}(\bmod A)$ be a tilting complex. Then:

(a) For every indecomposable direct summand $X$ of $T$ there exists $\widetilde{X} \in \mathcal{D}^{b}(\bmod \mathcal{C})$ (necessarily indecomposable) such that $F_{\lambda} \widetilde{X} \simeq X$.

Moreover, the class $\left\{\widetilde{X} \in \mathcal{D}^{b}(\bmod \mathcal{C}) \mid F_{\lambda} \widetilde{X}\right.$ is an indecomposable direct summand of $T\}$ satisfies the following:

(b) It generates the triangulated category $\mathcal{D}^{b}(\bmod \mathcal{C})$.

(c) It is stable under the action of $G$.

(d) $\mathcal{D}^{b}(\bmod \mathcal{C})\left(\widetilde{X},{ }^{g} \widetilde{Y}[i]\right)=0$ for every $\tilde{X}, \tilde{Y}$ in this class, $i \neq 0$ and $g \in G$.

We need the two following dual lemmas.

Lemma 4.2. Let $\Delta: X \stackrel{u}{\rightarrow} M \rightarrow Y \rightarrow X[1]$ be a triangle in $\mathcal{D}^{b}(\bmod A)$ such that:

(a) There exists $\tilde{X} \in \mathcal{D}^{b}(\bmod \mathcal{C})$ satisfying $X=F_{\lambda} \widetilde{X}$.

(b) $M=M_{1} \oplus \cdots \oplus M_{t}$ where $M_{1}, \ldots, M_{t}$ are indecomposables such that there exist indecomposable objects $\widetilde{M}_{1}, \ldots, \widetilde{M}_{t}$ satisfying $F_{\lambda} \widetilde{M}_{i}=M_{i}$ for every $i$.

(c) $\mathcal{D}^{b}(\bmod A)(Y, M[1])=0$. 
Then $\Delta$ is isomorphic to a triangle in $\mathcal{D}^{b}(\bmod A)$ :

$$
X \stackrel{\left[\begin{array}{lll}
F_{\lambda} u_{1}^{\prime} & \ldots & F_{\lambda} u_{t}^{\prime}
\end{array}\right]^{t}}{\longrightarrow} M_{1} \oplus \cdots \oplus M_{t} \rightarrow Y \rightarrow X[1]
$$

where $u_{i}^{\prime} \in \mathcal{D}^{b}(\bmod \mathcal{C})\left(\widetilde{X},{ }^{g_{i}} \widetilde{M}_{i}\right)$ for some $g_{i} \in G$ for every $i$.

Lemma 4.3. Let $\Delta: X \rightarrow M \stackrel{v}{\rightarrow} Y \rightarrow X[1]$ be a triangle in $\mathcal{D}^{b}(\bmod A)$ such that:

(a) There exists $\tilde{Y} \in \mathcal{D}^{b}(\bmod \mathcal{C})$ satisfying $Y=F_{\lambda} \tilde{Y}$.

(b) $M=M_{1} \oplus \cdots \oplus M_{t}$ where $M_{1}, \ldots, M_{t}$ are indecomposables such that there exist indecomposable objects $\widetilde{M}_{1}, \ldots, \widetilde{M}_{t}$ satisfying $F_{\lambda} \widetilde{M}_{i}=M_{i}$ for every $i$.

(c) $\mathcal{D}^{b}(\bmod A)(M, X[1])=0$.

Then $\Delta$ is isomorphic to a triangle in $\mathcal{D}^{b}(\bmod A)$ :

$$
X \rightarrow M_{1} \oplus \cdots \oplus M_{t} \stackrel{\left[\begin{array}{lll}
F_{\lambda} v_{1}^{\prime} & \cdots & F_{\lambda} v_{t}^{\prime}
\end{array}\right]}{\longrightarrow} Y \rightarrow X[1]
$$

where $v_{i}^{\prime} \in \mathcal{D}^{b}(\bmod \mathcal{C})\left({ }^{g_{i}} \widetilde{M}_{i}, \widetilde{Y}\right)$ for some $g_{i} \in G$ for every $i$.

Proof of 4.2. We say that a morphism $u \in \mathcal{D}^{b}(\bmod A)\left(X, M_{i}\right)$ is homogeneous of degree $g \in G$ if and only if there exists $u^{\prime} \in \mathcal{D}^{b}(\bmod \mathcal{C})\left(\widetilde{X},{ }^{g} \widetilde{M}_{i}\right)$ such that $u=F_{\lambda}\left(u^{\prime}\right)$. Since $F_{\lambda}: \mathcal{D}^{b}(\bmod \mathcal{C}) \rightarrow \mathcal{D}^{b}(\bmod A)$ has the covering property, any morphism $X \rightarrow M_{i}$ is (uniquely) the sum of $d$ nonzero homogeneous morphisms of pairwise different degrees (with $d \geqslant 0$ ). Let $u=\left[u_{1} \ldots u_{t}\right]^{t}$ with $u_{i}: X \rightarrow M_{i}$ for each $i$. We may assume that $u_{1}: X \rightarrow M_{1}$ is not homogeneous. Thus $u_{1}=h_{1}+\cdots+$ $h_{d}$, where $d \geqslant 2$ and $h_{1}, \ldots, h_{d}: X \rightarrow M_{1}$ are nonzero homogeneous morphisms of pairwise different degrees. In order to prove the lemma, it suffices to prove that $\Delta$ is isomorphic to a triangle $X \stackrel{u^{\prime}}{\rightarrow} M \rightarrow Y \rightarrow X[1]$ with $u^{\prime}=\left[u_{1}^{\prime} u_{2} \ldots u_{t}\right]^{t}$ such that $u_{1}^{\prime}$ is equal to the sum of at most $d-1$ nonzero homogeneous morphisms $X \rightarrow M_{1}$ of pairwise different degrees. For simplicity we adopt the following notation:

(1) $\bar{M}=M_{2} \oplus \cdots \oplus M_{t}$ (so $\left.M=M_{1} \oplus \bar{M}\right)$.

(2) $\bar{u}=\left[u_{2} \ldots u_{t}\right]^{t}: X \rightarrow \bar{M}$ (so $\left.u=\left[\begin{array}{ll}u_{1} & \bar{u}\end{array}\right]^{t}: X \rightarrow M_{1} \oplus \bar{M}\right)$.

(3) $\bar{h}=h_{2}+\cdots+h_{d}: X \rightarrow M_{1}$ (so $\left.u_{1}=h_{1}+\bar{h}\right)$.

Applying the functor $\mathcal{D}^{b}(\bmod A)\left(-, M_{1}\right)$ to $\Delta$ gives the exact sequence:

$$
\begin{aligned}
\mathcal{D}^{b}(\bmod A)\left(M_{1} \oplus \bar{M}, M_{1}\right) & \stackrel{\operatorname{Hom}\left(u, M_{1}\right)}{\longrightarrow} \mathcal{D}^{b}(\bmod A)\left(X, M_{1}\right) \\
& \rightarrow \mathcal{D}^{b}(\bmod A)\left(Y, M_{1}[1]\right)=0 .
\end{aligned}
$$

So there exists $[\lambda, \mu]: M_{1} \oplus \bar{M} \rightarrow M_{1}$ such that $h_{1}=[\lambda, \mu] u$. Hence,

$$
h_{1}=\lambda u_{1}+\mu \bar{u}=\lambda h_{1}+\lambda \bar{h}+\mu \bar{u} .
$$

We distinguish two cases according to whether $\lambda \in \operatorname{End}_{\mathcal{D}^{b}(\bmod A)}\left(M_{1}, M_{1}\right)$ is invertible or nilpotent. If $\lambda$ is invertible, then the following is an isomorphism in $\mathcal{D}^{b}(\bmod A)$ :

$$
\theta:=\left[\begin{array}{cc}
\lambda & \mu \\
0 & \operatorname{Id}_{\bar{M}}
\end{array}\right]: M_{1} \oplus \bar{M} \rightarrow M_{1} \oplus \bar{M}
$$


Using $(i)$ we deduce an isomorphism of triangles:

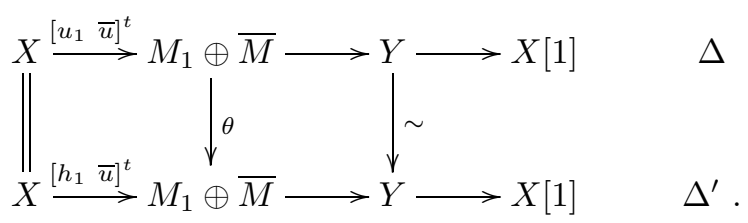

Since $h_{1}: X \rightarrow M_{1}$ is homogeneous, $\Delta^{\prime}$ satisfies the our requirements. If $\lambda$ is nilpotent, let $p \geqslant 0$ be such that $\lambda^{p}=0$. Using $(i)$ we get the following equalities:

$$
\begin{aligned}
h_{1} & =\lambda^{2} h_{1}+\left(\lambda^{2}+\lambda\right) \bar{h}+\left(\lambda+\operatorname{ld}_{M_{1}}\right) \mu \bar{u} \\
\vdots & \vdots \\
h_{1} & =\lambda^{t} h_{1}+\left(\lambda^{t}+\lambda^{t-1}+\cdots+\lambda\right) \bar{h}+\left(\lambda^{t-1}+\cdots+\lambda+\operatorname{Id}_{M_{1}}\right) \mu \bar{u} \\
\vdots & \vdots \\
h_{1} & =\lambda^{p} h_{1}+\left(\lambda^{p}+\lambda^{p-1}+\cdots+\lambda\right) \bar{h}+\left(\lambda^{p-1}+\cdots+\lambda+\operatorname{ld}_{M_{1}}\right) \mu \bar{u} .
\end{aligned}
$$

Since $\lambda^{p}=0$ and $u_{1}=h_{1}+\bar{h}$ we infer that:

$$
u_{1}=\lambda^{\prime} \bar{h}+\lambda^{\prime} \mu \bar{u},
$$

where $\lambda^{\prime}$ is the invertible morphism $\operatorname{Id}_{M_{1}}+\lambda+\cdots+\lambda^{p-1} \in \operatorname{End}_{\mathcal{D}^{b}(\bmod A)}\left(M_{1}, M_{1}\right)$. So we have an isomorphism in $\mathcal{D}^{b}(\bmod A)$ :

$$
\theta:=\left[\begin{array}{cc}
\lambda^{\prime} & \lambda^{\prime} \mu \\
0 & \operatorname{ld} \\
\bar{M}
\end{array}\right]: M_{1} \oplus \bar{M} \rightarrow M_{1} \oplus \bar{M} .
$$

Consequently, we have an isomorphism of triangles:

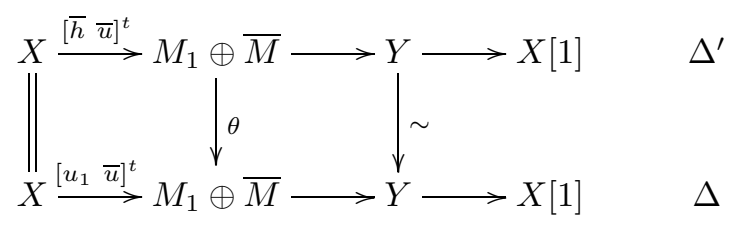

where $\bar{h}=h_{2}+\cdots+h_{p}$ is the sum of $p-1$ nonzero homogeneous morphisms of pairwise different degrees. So $\Delta^{\prime}$ satisfies our requirements.

The proof of 4.3 is the dual of the one of 4.2 so we omit it. Now we can prove 4.1 .

Proof of 4.1. If (a) holds true, then so does (c) because $F_{\lambda}: \mathcal{D}^{b}(\bmod \mathcal{C}) \rightarrow \mathcal{D}^{b}(\bmod A)$ is $G$-invariant. Recall that $\Theta: \mathcal{D}^{b}(\mathcal{H}) \rightarrow \mathcal{D}^{b}(\bmod A)$ is a triangle equivalence. As in Section 3] we write $\mathcal{T}$ (or $\mathcal{T}^{\prime}$ ) for the set of isomorphism classes of objects $T \in \mathcal{D}^{b}(\mathcal{H})$ (or $\left.T \in \mathcal{D}^{b}(\bmod A)\right)$ such that $T$ is the direct sum of $n$ pairwise nonisomorphic indecomposable objects and $\mathcal{D}^{b}(\mathcal{H})(T, T[i])=0\left(\operatorname{or} \mathcal{D}^{b}(\bmod A)(T, T[i])=0\right.$, respectively) for every $i \geqslant 1$. Therefore:

(i) $\Theta$ defines a bijection $\Theta: \mathcal{T} \rightarrow \mathcal{T}^{\prime}$. Under this bijection, tilting complexes in $\mathcal{D}^{b}(\mathcal{H})$ correspond to tilting complexes in $\mathcal{D}^{b}(\bmod A)$. 
We prove that (a) and (b) hold true for any $T \in \mathcal{T}^{\prime}$ (and therefore for any tilting object in $\left.\mathcal{D}^{b}(\bmod A)\right)$. For this purpose, we use the results of Section 3 First of all, remark that:

(ii) The assertions (a) and (b) hold true for $T=A$. In this case, $F_{\lambda} \widetilde{X}$ is an indecomposable summand of $A$ if and only if $\widetilde{X}$ is an indecomposable projective $\mathcal{C}$-module.

If $\widetilde{X} \in \mathcal{D}^{b}(\mathcal{H})$, then $F_{\lambda}(\widetilde{X}[1])=\left(F_{\lambda} \widetilde{X}\right)[1]$. Therefore:

(iii) Let $T, T^{\prime} \in \mathcal{T}^{\prime}$ be such that $\Theta^{-1}\left(T^{\prime}\right)$ and $\Theta^{-1}(T)$ are related by a transformation of the first kind. Then (a) and (b) hold true for $T$ if and only if they do so for $T^{\prime}$.

Now assume that $T, T^{\prime} \in \mathcal{T}^{\prime}$ are such that $\Theta^{-1}\left(T^{\prime}\right)$ is obtained from $\Theta^{-1}(T)$ by a transformation of the second kind. We prove that (a) and (b) hold true for $T$ if and only if they do so for $T^{\prime}$. In such a situation there exist $X, Y \in \mathcal{D}^{b}(\bmod A)$ indecomposables and $\bar{T} \in \mathcal{D}^{b}(\bmod A)$ such that $T=X \oplus \bar{T}$ and $T^{\prime}=Y \oplus \bar{T}$. Also, there exists a triangle in $\mathcal{D}^{b}(\bmod A)$ of one the two following forms:

(1) $X \rightarrow M \rightarrow Y \rightarrow X[1]$ where $M \in \operatorname{add}(\bar{T})$.

(2) $Y \rightarrow M \rightarrow X \rightarrow Y[1]$ where $M \in \operatorname{add}(\bar{T})$.

Assume that (a) and (b) hold true for $T$ and that there is a triangle $X \rightarrow M \rightarrow$ $Y \rightarrow X[1]$ (the other cases are dealt with using similar arguments). In order to prove that (a) and (b) hold true for $T^{\prime}$ we prove that $Y \simeq F_{\lambda} \widetilde{Y}$ for some $\tilde{Y} \in \mathcal{D}^{b}(\bmod \mathcal{C})$. Fix an indecomposable decomposition $M=M_{1} \oplus \cdots \oplus M_{t}$. By assumption on $T$, there exist indecomposable objects $\widetilde{X}, \widetilde{M}_{1}, \ldots, \widetilde{M}_{t} \in \mathcal{D}^{b}(\bmod \mathcal{C})$ such that $F_{\lambda} \widetilde{X} \simeq X, F_{\lambda} \widetilde{M}_{1} \simeq M_{1}, \ldots, F_{\lambda} \widetilde{M}_{t} \simeq M_{t}$. Using these isomorphisms, we identify $F_{\lambda} \widetilde{X}$ and $F_{\lambda} \widetilde{M}_{i}$ to $X$ and $M_{i}$, respectively. By 4.2 , there exist $g_{1}, \ldots, g_{t} \in G$ and morphisms $u_{i} \in \mathcal{D}^{b}(\bmod \mathcal{C})\left(\widetilde{X},{ }^{g_{i}} \widetilde{M}_{i}\right)$ (for $\left.i \in\{1, \ldots, t\}\right)$ such that the triangle $X \rightarrow M \rightarrow Y \rightarrow X[1]$ is isomorphic to a triangle of the form:

$$
X \stackrel{\left[\begin{array}{lll}
F_{\lambda}\left(u_{1}\right) & \ldots & F_{\lambda}\left(u_{t}\right)
\end{array}\right]^{t}}{\longrightarrow} M \rightarrow Y \rightarrow X[1] .
$$

Set $u=\left[u_{1} \cdots u_{t}\right]^{t}: \widetilde{X} \rightarrow{ }^{g_{1}} \widetilde{M}_{1} \oplus \cdots \oplus{ }^{g_{t}} \widetilde{M}_{t}$. We complete $u$ into a triangle $\widetilde{X} \stackrel{u}{\rightarrow}{ }^{g_{1}} \widetilde{M}_{1} \oplus \cdots \oplus{ }^{g_{t}} \widetilde{M}_{t} \stackrel{v}{\rightarrow} \tilde{X}[1]$ in $\mathcal{D}^{b}(\bmod \mathcal{C})$. So we have a triangle $X \stackrel{F_{\lambda}(u)}{\longrightarrow}$ $M \stackrel{F_{\lambda}(v)}{\longrightarrow} F_{\lambda} \tilde{Y} \rightarrow X[1]$ in $\mathcal{D}^{b}(\bmod A)$. Therefore, $Y \simeq F_{\lambda} \tilde{Y}$. So (a) holds true for $T^{\prime}$ and the class $\left\{\widetilde{Z} \mid F_{\lambda} \widetilde{Z}\right.$ is an indecomposable direct summand of $\left.T^{\prime}\right\}$ coincides with the class $\left\{{ }^{g} \widetilde{Y} \mid g \in G\right\} \cup\left\{\widetilde{Z} \mid F_{\lambda} \widetilde{Z}\right.$ is an indecomposable direct summand of $\left.\bar{T}\right\}$ (see 2.3). Because (b) holds true for $T$ and because of the triangle $\widetilde{X} \rightarrow{ }^{g_{1}} \widetilde{M}_{1} \oplus$ $\cdots \oplus{ }^{g_{t}} \widetilde{M}_{t} \rightarrow \widetilde{Y} \rightarrow \widetilde{X}[1]$ we deduce that (b) holds for $T^{\prime}$. So we have proved the following:

(iv) Let $T, T^{\prime} \in \mathcal{T}^{\prime}$ be such that $\Theta^{-1}\left(T^{\prime}\right)$ is obtained from $\Theta^{-1}(T)$ by a transformation of the second kind. Then (a) and (b) hold true for $T$ if and only if they do so for $T^{\prime}$.

By 3.7. (i), (ii), (iii) and (iv) the assertions (a), (b) and (c) are satisfied for any $T \in \mathcal{T}$. Finally, if $T$ is a tilting complex, then (d) follows from the fact that $\mathcal{D}^{b}(\bmod A)(T, T[i])=0$ for every $i \neq 0$ and from 2.1.

It is interesting to note that the transformations of the second kind in $\mathcal{D}^{b}(\bmod A)$ give rise to transformations of the second kind in $\mathcal{D}^{b}(\bmod \mathcal{C})$. Indeed, let $T, T^{\prime}$ be 
in $\mathcal{T}^{\prime}$ where $\mathcal{T}^{\prime}$ is as in the proof of 4.1. Assume that $T=M \oplus \bar{T}$ with $M$ indecomposable, $T=M^{*} \oplus \bar{T}$ with $M^{*}$ indecomposable and there exists a triangle $\Delta: M \rightarrow B \rightarrow M^{*} \rightarrow M[1]$ in $\mathcal{D}^{b}(\bmod A)$ where $M \rightarrow B\left(\right.$ or $\left.B \rightarrow M^{*}\right)$ is a left minimal add $(\bar{T})$-approximation of $M$ (or a right minimal add $(\bar{T})$-approximation of $M^{*}$, respectively). Then the following holds.

Lemma 4.4. Keep the above setting. Let $B=\bigoplus_{i=1}^{t} B_{i}$ be an indecomposable decomposition (maybe with multiplicities). Then there exists a triangle $\widetilde{\Delta}: \widetilde{M} \stackrel{u}{\rightarrow}$ $\bigoplus_{i=1}^{t}{ }^{g_{i}} \widetilde{B}_{i} \stackrel{v}{\rightarrow}{ }^{g_{0}} \widetilde{M}^{*} \rightarrow M[1]$ in $\mathcal{D}^{b}(\bmod \mathcal{C})$ whose image under $F_{\lambda}$ is isomorphic to $\Delta$. Moreover, if $\mathcal{X}\left(\right.$ or $\left.\mathcal{X}^{\prime}\right)$ denotes the additive full subcategory of $\mathcal{D}^{b}(\bmod \mathcal{C})$ generated by the indecomposables $X \in \mathcal{D}^{b}(\bmod \mathcal{C})$ not isomorphic to $\widetilde{M}$ (or to $\widetilde{M}^{*}$ ) and such that $F_{\lambda} X$ is an indecomposable summand of $T$ (or of $T^{\prime}$, respectively), then:

(a) $u$ is a left minimal $\mathcal{X}$-approximation,

(b) $v$ is a right minimal $\mathcal{X}^{\prime}$-approximation.

Proof. For every $X \in \operatorname{add}(\bar{T})$ indecomposable we fix $\widetilde{X} \in \mathcal{D}^{b}(\bmod \mathcal{C})$ such that $F_{\lambda} \widetilde{X}=X$. So $\mathcal{X}=\left\{{ }^{g} \widetilde{M} \mid g \in G \backslash\{1\}\right\} \cup\left\{{ }^{g} \widetilde{X} \mid X \in\right.$ add $(\bar{T})$ indecomposable, $\left.g \in G\right\}$. The existence of $\widetilde{\Delta}$ follows from the proof of 4.1 . So $F_{\lambda}(u)$ is a left minimal add $(\bar{T})$ approximation. This and the exactness of $F_{\lambda}$ imply that $u$ is left minimal. Let $f: \widetilde{M} \rightarrow{ }^{g} \widetilde{Y}$ be a nonzero morphism where ${ }^{g} \widetilde{Y} \in \mathcal{X}$ and $Y \in \operatorname{add}(T)$ indecomposable. The linear map $\bigoplus_{h \in G} \mathcal{D}^{b}(\bmod \mathcal{C})\left(\widetilde{M},{ }^{h} \widetilde{M}\right) \rightarrow \operatorname{End}_{\mathcal{D}^{b}(\bmod A)}(M, M)$ induced by $F_{\lambda}$ is bijective. Also, $\operatorname{dim}_{k} \operatorname{End}_{\mathcal{D}^{b}(\bmod A)}(M, M)=1$ because $M$ is indecomposable and $\mathcal{D}^{b}(\bmod A)(M, M[i])=0$ for every $i>0$. So ${ }^{g} \widetilde{Y} \not{ }^{h} \widetilde{M}$ for every $h \neq 1$. Hence, $Y \in \operatorname{add}(\bar{T})$ and, therefore, $F_{\lambda}(f)$ factors through $F_{\lambda}(u)$ :

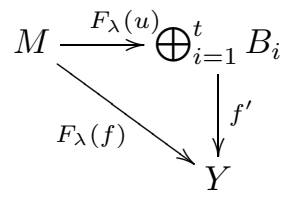

There exists $\left(f_{h}^{\prime}\right)_{h} \in \bigoplus_{h \in G} \mathcal{D}^{b}(\bmod \mathcal{C})\left(\bigoplus_{i=1}^{t}{ }^{g_{i}} \widetilde{B}_{i},{ }^{h} \widetilde{Y}\right)$ such that $f^{\prime}=\sum_{h \in G} F_{\lambda}\left(f_{h}^{\prime}\right)$ because of the covering property of $F_{\lambda}$. So $F_{\lambda}(f)=\sum_{h \in G} F_{\lambda}\left(f_{h}^{\prime} u\right)$ and, therefore, $f=f_{g}^{\prime} u$ for the same reason. Thus $u$ is a left minimal $\mathcal{X}$-approximation. Similarly, $v$ is a right minimal $\mathcal{X}^{\prime}$-approximation.

Since tilting $A$-modules are particular cases of tilting complexes, we get the following result.

Corollary 4.5. Let $A$ be an algebra derived equivalent to a hereditary abelian category $\mathcal{H}$ such that $\overrightarrow{\mathcal{K}}_{\mathcal{H}}$ is connected. Let $F: \mathcal{C} \rightarrow A$ be a Galois covering with group $G$ where $\mathcal{C}$ is locally bounded, $T$ a tilting $A$-module and $X \in \bmod A$ an indecomposable summand of $T$. Then there exists $\widetilde{X} \in \bmod \mathcal{C}$ such that $F_{\lambda} \widetilde{X} \simeq X$.

Proof. By 4.1] such an $\tilde{X}$ exists in $\mathcal{D}^{b}(\bmod \mathcal{C})$. We prove that $\tilde{X}$ is isomorphic to a $\mathcal{C}$-module. Let $P \in \bmod \mathcal{C}$ be projective and $i \in \mathbb{Z} \backslash\{0\}$. Then $F_{\lambda} P \in \bmod A$ is projective and $\mathcal{D}^{b}(\bmod A)\left(F_{\lambda} P, X[i]\right)=0$ because $X$ is an $A$-module. On the other hand, the spaces $\mathcal{D}^{b}(\bmod A)\left(F_{\lambda} P, X[i]\right)$ and $\bigoplus_{g \in G} \mathcal{D}^{b}(\bmod \mathcal{C})\left({ }^{g} P, \tilde{X}[i]\right)$ are isomorphic. So $\mathcal{D}^{b}(\bmod \mathcal{C})(P, \widetilde{X}[i])=0$ for every $i \neq 0$. Thus, $\widetilde{X} \simeq H^{0}(\widetilde{X}) \in$ $\bmod \mathcal{C}$. 


\section{Proof of assertion $\left(\mathrm{H}_{2}\right)$.}

Proposition 4.6. Let $A$ be as in 4.5, $F: \mathcal{C} \rightarrow A$ a Galois covering with group $G$ where $\mathcal{C}$ is locally bounded and $X$ an indecomposable direct summand of a tilting complex in $\mathcal{D}^{b}(\bmod A)$. Assume that $F_{\lambda} \widetilde{X} \simeq X$ for some $\widetilde{X} \in \mathcal{D}^{b}(\bmod \mathcal{C})$. Then ${ }^{g} \widetilde{X} \nsucceq \widetilde{X}$ for every $g \in G \backslash\{1\}$.

Proof. We have $\operatorname{dim}_{k} \operatorname{End}_{\mathcal{D}^{b}(\bmod A)}(X, X)=1$ because $X$ is indecomposable and $\mathcal{D}^{b}(\bmod A)(X, X[i])=0$ for every $i \neq 0$. On the other hand, the spaces

$$
\bigoplus_{g \in G} \mathcal{D}^{b}(\bmod \mathcal{C})\left({ }^{g} \widetilde{X}, \widetilde{X}\right) \quad \text { and } \operatorname{End}_{\mathcal{D}^{b}(\bmod A)}(X, X)
$$

are isomorphic. So $\mathcal{D}^{b}(\bmod \mathcal{C})\left({ }^{g} \widetilde{X}, \widetilde{X}\right)=0$ and, therefore, ${ }^{g} \widetilde{X} \nsucceq X$ if $g \neq 1$.

Proof of assertion $\left(H_{3}\right)$. If $\psi: A \rightarrow A$ is an automorphism (and therefore a Galois covering with trivial group), then $\psi_{\lambda}: \bmod A \rightarrow \bmod A$ is an equivalence. It thus induces a triangle equivalence $\psi_{\lambda}: \mathcal{D}^{b}(\bmod A) \rightarrow \mathcal{D}^{b}(\bmod A)$.

Proposition 4.7. Let $A$ be as in 4.5, let $\psi: A \stackrel{\sim}{\rightarrow} A$ be an automorphism such that $\psi(x)=x$ for every $x \in \mathrm{ob}(A)$ and $T \in \mathcal{D}^{b}(\bmod A)$ a tilting complex. Then $\psi_{\lambda} X \simeq X$ in $\mathcal{D}^{b}(\bmod A)$ for every indecomposable summand $X$ of $T$.

Proof. Since $\psi(x)=x$ for every $x \in \mathrm{ob}(A)$, we have the following fact:

(i) The conclusion of the proposition holds true if $X$ is an indecomposable projective $A$-module.

Recall that $\Theta: \mathcal{D}^{b}(\mathcal{H}) \rightarrow \mathcal{D}^{b}(\bmod A)$ is a triangle equivalence. We keep the notation $\mathcal{T}$ and $\mathcal{T}^{\prime}$ introduced in the proof of 4.1 We prove the proposition for any $T \in \mathcal{T}^{\prime}$. By construction of $\Theta$, we have:

(ii) $\Theta$ induces a bijection $\Theta: \mathcal{T} \rightarrow \mathcal{T}^{\prime}$. Under this bijection, tilting complexes in $\mathcal{D}^{b}(\mathcal{H})$ correspond to tilting complexes in $\mathcal{D}^{b}(\bmod A)$.

Since $\psi_{\lambda}: \mathcal{D}^{b}(\bmod A) \rightarrow \mathcal{D}^{b}(\bmod A)$ is an equivalence, we also have:

(iii) Let $T, T^{\prime} \in \mathcal{T}$ be such that $T^{\prime}$ is obtained from $T$ by a transformation of the first kind. Then the proposition holds true for $T$ if and only if it does so for $T^{\prime}$.

Now assume that $T, T^{\prime} \in \mathcal{T}^{\prime}$ are such that $\Theta^{-1}\left(T^{\prime}\right)$ is obtained from $\Theta^{-1}(T)$ by a transformation of the second kind. We prove that the proposition holds true for $T$ if and only if it does so for $T^{\prime}$. There exist $X, Y \in \mathcal{D}^{b}(\bmod A)$ indecomposables and $\bar{T} \in \mathcal{D}^{b}(\bmod A)$ such that $T=X \oplus \bar{T}$ and $T^{\prime}=Y \oplus \bar{T}$. Also, there exists a triangle in $\mathcal{D}^{b}(\bmod A)$ of one of the two following forms:

(1) $X \rightarrow M \rightarrow Y \rightarrow X[1]$ where $X \rightarrow M$ is a left minimal add( $\bar{T})$-approximation and $M \rightarrow Y$ is a right minimal $\operatorname{add}(\bar{T})$-approximation.

(2) $Y \rightarrow M \rightarrow X \rightarrow Y[1]$ where $Y \rightarrow M$ is a left minimal add $(\bar{T})$-approximation and $M \rightarrow X$ is a right minimal add $(\bar{T})$-approximation.

Assume that the proposition holds true for $T$ and there is a triangle $X \rightarrow M \rightarrow$ $Y \rightarrow X[1]$ (the other cases are dealt with using similar arguments). We only need to prove that $\psi_{\lambda} Y \simeq Y$. Apply $\psi_{\lambda}$ to the triangle $X \rightarrow M \rightarrow Y \rightarrow X[1]$. Since $\psi_{\lambda}$ is an equivalence and the proposition holds true for $T$, there exists a triangle 
$X \rightarrow M \rightarrow \psi_{\lambda} Y \rightarrow X[1]$ in $\mathcal{D}^{b}(\bmod A)$ where $X \rightarrow M$ is a left minimal add( $\left.\bar{T}\right)$ approximation. Therefore, $\psi_{\lambda} Y \simeq Y$ in $\mathcal{D}^{b}(\bmod A)$. So we proved that:

(iv) If $T, T^{\prime} \in \mathcal{T}^{\prime}$ are such that $\Theta^{-1}\left(T^{\prime}\right)$ is obtained from $\Theta^{-1}(T)$ by a transformation of the second kind, then the proposition holds true for $T$ if and only if it does so for $T^{\prime}$.

As in the proof of 4.1 the conclusion follows from $(i),(i i),(i i i),(i v)$ and 3.7

Application: Proof of Theorem [C. We prove Theorem [C] as an application of the preceding results. We need the following lemma. If $T=T_{1} \oplus \cdots \oplus T_{n} \in$ $\mathcal{D}^{b}(\bmod A)$ is an indecomposable decomposition of a multiplicity-free complex $T$, then $\operatorname{End}_{\mathcal{D}^{b}(\bmod A)}(T)$ is naturally a locally bounded $k$-category, equal to the full subcategory of $\mathcal{D}^{b}(\bmod A)$ with object $T_{1}, \ldots, T_{n}$.

Lemma 4.8. Let $A$ be a piecewise hereditary algebra of type $Q$. Let $F: \mathcal{C} \rightarrow A$ be a connected Galois covering with group $G$. Let $T \in \mathcal{D}^{b}(\bmod A)$ be a tilting complex, $B=\operatorname{End}_{\mathcal{D}^{b}(\bmod A)}(T)$ and $T=T_{1} \oplus \cdots \oplus T_{n}$ an indecomposable decomposition. Let $\lambda_{i}: F_{\lambda} \widetilde{T}_{i} \rightarrow T_{i}$ be an isomorphism where $\widetilde{T}_{i} \in \mathcal{D}^{b}(\bmod \mathcal{C})$ is indecomposable for every $i$. This defines the bounded complex of (not necessarily finite dimensional) $\mathcal{C}$-modules $\widetilde{T}:=\bigoplus_{i, g}{ }^{g} \widetilde{T}_{i}$, where the sum runs over $g \in G$ and $i \in\{1, \ldots, n\}$. Let $\mathcal{C}^{\prime}$ be the full subcategory of $\mathcal{D}^{b}(\bmod \mathcal{C})$ with objects the complexes ${ }^{g} \widetilde{T}_{i}$ (for $g \in G, i \in\{1, \ldots, n\})$. Then the triangle functor $F_{\lambda}: \mathcal{D}^{b}(\bmod \mathcal{C}) \rightarrow \mathcal{D}^{b}(\bmod A)$ induces a connected Galois covering with group $G$ :

$$
\begin{aligned}
& F_{\widetilde{T}, \lambda}: \mathcal{C}^{\prime} \rightarrow B, \\
&{ }^{g} \widetilde{T}_{i}, \mapsto T_{i}, \\
&{ }^{g} \widetilde{T}_{i} \stackrel{u}{\rightarrow}{ }^{h} \widetilde{T}_{j} \mapsto T_{i} \stackrel{\lambda_{j} F_{\lambda}(u) \lambda_{i}^{-1}}{\longrightarrow} T_{j} .
\end{aligned}
$$

The complex $\widetilde{T}$ is naturally a bounded complex of $\mathcal{C}^{\prime}-\mathcal{C}$-bimodules: As a functor from $\mathcal{C}^{\prime} \times \mathcal{C}^{o p}$ to $\mathrm{MOD} k$, it assigns the vector space ${ }^{g} \widetilde{T}_{i}(x)$ to the pair of objects $\left({ }^{g} \widetilde{T}_{i}, x\right)$. The total derived functor:

$$
-\underset{\mathcal{C}^{\prime}}{\mathbb{L}} \widetilde{T}: \mathcal{D}^{b}\left(\bmod \mathcal{C}^{\prime}\right) \rightarrow \mathcal{D}^{b}(\bmod \mathcal{C})
$$

is a $G$-equivariant triangle equivalence. Finally, if $T$ is a tilting $A$-module and all the objects $\widetilde{T}_{i}$ are $\mathcal{C}$-modules (see 4.5), then:

(a) $\operatorname{Ext}_{\mathcal{C}}^{1}\left({ }^{g} \widetilde{T}_{i},{ }^{h} \widetilde{T}_{j}\right)=0$ for every $i, j \in\{1, \ldots, n\}$ and $g, h \in G$.

(b) $\operatorname{pd}_{\mathcal{C}}\left({ }^{g} \widetilde{T}_{i}\right) \leqslant 1$ for every $i, g$.

(c) If $P \in \bmod \mathcal{C}$ is an indecomposable projective $\mathcal{C}$-module, then there exists an exact sequence $0 \rightarrow P \rightarrow T^{(1)} \rightarrow T^{(2)} \rightarrow 0$ in $\bmod \mathcal{C}$ where $T^{(1)}, T^{(2)} \in$ $\operatorname{add}\left(\left\{{ }^{g} \widetilde{T}_{i} \mid i \in\{1, \ldots, n\}, g \in G\right\}\right)$.

Proof. By 2.1 the functor $F_{\widetilde{T}, \lambda}$ is a well-defined Galois covering. By 4.6, we know that $\mathcal{C}^{\prime}$ is a locally bounded $k$-category (see [33, 2.1], for more details on the construction of $\left.F_{\widetilde{T}, \lambda}\right)$. We prove that $\mathcal{C}^{\prime}$ is connected. By definition of $\widetilde{T}$ we have ${ }^{g} \widetilde{T}=\widetilde{T}$ for every $g \in G$. Hence the functor $-\underset{\mathcal{C}^{\prime}}{\mathbb{L}} \widetilde{T}$ is $G$-equivariant. On the 
other hand, $-{\underset{\mathcal{C}}{\mathcal{C}^{\prime}}}_{\mathbb{L}}^{\mathbb{T}} \widetilde{T}$ is a triangle equivalence. Indeed, by $4.1(\mathrm{~d})$, and by classical arguments on derived equivalences (see 19, III.2], for instance), this functor is full and faithful. Moreover, its image contains the complexes ${ }^{g} \widetilde{T}_{i}$ (for $g \in G$ and $i \in\{1, \ldots, n\})$. So 4.1 (b), implies that this functor is dense and, therefore, is a triangle equivalence $\mathcal{D}^{b}\left(\bmod \mathcal{C}^{\prime}\right) \rightarrow \mathcal{D}^{b}(\bmod \mathcal{C})$. In particular, $\mathcal{C}^{\prime}$ is connected. So $F_{\widetilde{T}, \lambda}: \mathcal{C}^{\prime} \rightarrow B$ is a connected Galois covering with group $G$ and $-\underset{\mathcal{C}^{\prime}}{\mathbb{L}} \widetilde{T}$ is a $G$-equivariant triangle equivalence.

Now we assume that $T$ is a tilting $A$-module. Assertion (a) follows from 4.1(d). Assertion (b) follows from the fact that $\operatorname{pd}_{A}(T) \leqslant 1$ and $F_{\lambda}: \bmod \mathcal{C} \rightarrow \bmod A$ is exact and faithful. We prove assertion (c). Let $P \in \bmod \mathcal{C}$ be indecomposable projective. Since $F_{\lambda} P$ is projective, there exists an exact sequence $0 \rightarrow F_{\lambda} P \rightarrow$ $X \rightarrow Y \rightarrow 0$ in $\bmod A$ with $X, Y \in \operatorname{add}(T)$. By 4.2, the triangle $F_{\lambda} P \rightarrow X \rightarrow Y \rightarrow$ $F_{\lambda} P[1]$ is isomorphic to the image under $F_{\lambda}$ of a triangle $P \rightarrow X^{\prime} \rightarrow Y^{\prime} \rightarrow P[1]$ where $X^{\prime}, Y^{\prime} \in \operatorname{add}\left(\left\{{ }^{g} \widetilde{T}_{i} \mid g \in G, i \in\{1, \ldots, n\}\right\}\right)$. Since $F_{\lambda}$ is exact and faithful, the sequence $0 \rightarrow P \rightarrow X^{\prime} \rightarrow Y^{\prime} \rightarrow 0$ is an exact sequence in $\bmod \mathcal{C}$.

For convenience, we write $\operatorname{End}_{\mathcal{D}^{b}(\bmod \mathcal{C})}(\widetilde{T})$ for the category $\mathcal{C}^{\prime}$ of 4.8 .

Remark 4.9. Keep the hypotheses and notation of the preceding lemma. If $G$ is finite and if $T$ is a tilting $A$-module, then the lemma expresses that $\bigoplus_{g, i}{ }^{g} \widetilde{T}_{i}$ is a tilting $\mathcal{C}$-module.

Now we can prove Theorem $\mathrm{C}$ which was stated in the introduction.

Proof of Theorem C. By [19, Cor. 5.5], there exists a sequence of algebras:

$$
A_{0}=k Q, A_{1}=\operatorname{End}_{A_{0}}\left(T^{(0)}\right), \ldots, A_{l}=\operatorname{End}_{A_{l-1}}\left(T^{(l-1)}\right)=A
$$

such that $T^{(i)} \in \bmod A_{l-1}$ is tilting for every $i$. We prove the theorem by induction on $l$. If $l=0$, then $A=k Q$. For any connected Galois covering $\mathcal{C} \rightarrow A$ with group $G$ there exists a Galois covering of quivers $Q^{\prime} \rightarrow Q$ with group $G$ such that $\mathcal{C} \simeq k Q^{\prime}$ ([32, Prop. 4.4]). Assume that $l>0$ and the conclusion of the theorem holds true for $A_{l-1}$. Let $\mathcal{C} \rightarrow A$ be a connected Galois covering with group $G$. Note that $T^{(l-1)}$ is a tilting $A^{o p}$-module. Applying the preceding lemma to the Galois covering $\mathcal{C}^{o p} \rightarrow A^{o p}$ with group $G$ yields a connected Galois covering $\mathcal{C}^{\prime} \rightarrow \operatorname{End}_{A^{o p}}\left(T^{(l-1)}\right)$ with group $G$ such that $\mathcal{D}^{b}\left(\bmod \mathcal{C}^{o p}\right)$ and $\mathcal{D}^{b}\left(\bmod \mathcal{C}^{\prime}\right)$ are triangle equivalent. On the other hand, $A_{l-1} \simeq \operatorname{End}_{A^{o p}}\left(T^{(l-1)}\right)^{o p}$. Therefore, the induction hypothesis implies that $\mathcal{D}^{b}\left(\bmod \mathcal{C}^{\prime o p}\right)$ is triangle equivalent to $\mathcal{D}^{b}\left(\bmod k Q^{\prime}\right)$ where $Q^{\prime}$ is a quiver such that there exists a Galois covering of quivers $Q^{\prime} \rightarrow Q$ with group $G$.

Remark 4.10. Let $A$ be a finite dimensional algebra endowed with a (not necessarily free) $G$-action. Then:

(a) If the $G$-action on $A$ is free, then the quotient algebra $A / G$ is well defined. The proof of Theorem $\mathrm{C}$ shows that if $A / G$ is tilted (or, more generally, piecewise hereditary of type a quiver), then so is $A$.

(b) It is proved in [13, Thm. 3] that if the order of $G$ is invertible in $k$ and if $A$ is piecewise hereditary, then so is the skew-group algebra $A[G]$. Recall that if the finite group $G$ acts freely on $A$, then the algebras $A[G]$ and $A / G$ are Morita equivalent ([12, Thm.2.8]). 


\section{Correspondence Between Galois Coverings}

We still assume that $A$ is derived equivalent to a hereditary abelian category $\mathcal{H}$ such that $\overrightarrow{\mathcal{K}}_{\mathcal{H}}$ is connected. Let $T \in \mathcal{D}^{b}(\bmod A)$ be a tilting complex and $B=\operatorname{End}_{\mathcal{D}^{b}(\bmod A)}(T)$. In this section we construct a correspondence between the Galois coverings of $A$ and those of $B$. This work has been done in $[33$, in the particular case where $T$ is a tilting $A$-module. In order to compare the Galois coverings of $A$ and those of $B$, it is convenient to use the notion of equivalent Galois covering. Given two Galois coverings $F: \mathcal{C} \rightarrow A$ and $F^{\prime}: \mathcal{C}^{\prime} \rightarrow A$ we say that $F$ and $F^{\prime}$ are equivalent if there exists a commutative diagram:

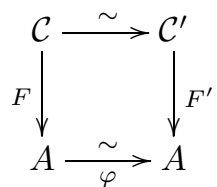

where the horizontal arrows are isomorphisms and $\varphi: A \rightarrow A$ is an automorphism such that $\varphi(x)=x$ for every $x \in \mathrm{ob}(A)$.

Equivalence classes of Galois coverings of $A$ associated to equivalence classes of Galois coverings of $B$. In 4.8, we have associated a Galois covering $F_{\widetilde{T}, \lambda}$ of $B$ to any Galois covering of $A$ and to any data consisting of isomorphisms $\left(\lambda_{i}: F_{\lambda} \widetilde{T}_{i} \rightarrow T_{i}\right)_{i=1, \ldots, n}$ in $\mathcal{D}^{b}(\bmod \mathcal{C})$. The following lemma shows that different choices for these data give rise to equivalent Galois coverings.

Lemma 5.1 ([33, $\S 2])$. Let $F: \mathcal{C} \rightarrow A$ be a connected Galois covering with group $G$. Let $T \in \mathcal{D}^{b}(\bmod A)$ be a tilting complex and $T=T_{1} \oplus \cdots \oplus T_{n}$ be an indecomposable decomposition.

(a) Let $\left(\lambda_{i}: F_{\lambda} \widetilde{T}_{i} \rightarrow T_{i}\right)_{i=1, \ldots, n}$ and $\left(\mu_{i}: F_{\lambda} \widehat{T}_{i} \rightarrow T_{i}\right)_{i=1, \ldots, n}$ be isomorphisms in $\mathcal{D}^{b}(\bmod A)$ defining the Galois coverings $F_{\widetilde{T}, \lambda}: \operatorname{End}_{\mathcal{D}^{b}(\bmod \mathcal{C})}(\widetilde{T}) \rightarrow$ $\operatorname{End}_{\mathcal{D}^{b}(\bmod A)}(T)$ and $F_{\widehat{T}, \mu}: \operatorname{End}_{\mathcal{D}^{b}(\bmod \mathcal{C})}(\widehat{T}) \rightarrow \operatorname{End}_{\mathcal{D}^{b}(\bmod A)}(T)$, respectively. Then $F_{\widetilde{T}, \lambda}$ and $F_{\widehat{T}, \mu}$ are equivalent. We write $[F]_{T}$ for the corresponding equivalence class of Galois coverings of $\operatorname{End}_{\mathcal{D}^{b}(\bmod A)}(T)$.

(b) Let $F^{\prime}: \mathcal{C}^{\prime} \rightarrow A$ be a connected Galois covering with group $G$ and equivalent to $F$. Then the equivalence classes $[F]_{T}$ and $\left[F^{\prime}\right]_{T}$ coincide.

Proof. In the case of tilting modules, (a) and (b) were proved in [33, Lem. 2.4] and [33. Lem. 2.5], respectively. Using 2.3 and 4.7, it is easily checked that the same arguments apply to prove (a) and (b) in the present situation.

In the sequel we keep the notation $[F]_{T}$ introduced in 5.1 .

Galois coverings of $A$ induced by Galois coverings of $B$. We now express any Galois covering of $A$ as induced by a Galois covering of $B$ as in 4.8 , The tilting complex $T$ is naturally a complex of $B-A$-bimodules. Also, it defines a triangle equivalence:

$$
-{\underset{B}{\mathbb{L}}}_{B}^{\mathbb{L}} X: \mathcal{D}^{b}(\bmod B) \rightarrow \mathcal{D}^{b}(\bmod A)
$$


Fix a connected Galois covering $F: \mathcal{C} \rightarrow A$ with group $G$, an indecomposable decomposition $T=T_{1} \oplus \cdots \oplus T_{n}$ and isomorphisms $\left(\mu_{i}: F_{\lambda} \widetilde{T}_{i} \stackrel{\sim}{\longrightarrow} T_{i}\right)_{i=1, \ldots, n}$. According to 4.8, these data define the Galois covering $F_{\widetilde{T}, \mu}: \operatorname{End}_{\mathcal{D}^{b}(\bmod \mathcal{C})}(\widetilde{T}) \rightarrow B$ which we denote by $F^{\prime}: \mathcal{C}^{\prime} \rightarrow B$ for simplicity.

Lemma 5.2. The following diagram commutes up to an isomorphism of functors:

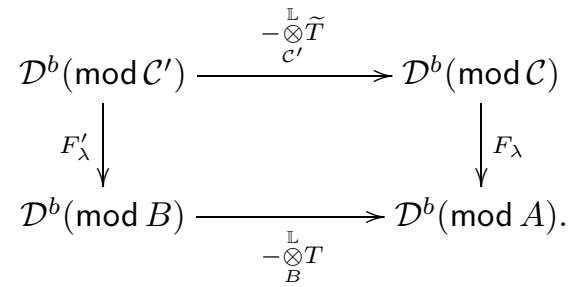

Proof. Recall that $F_{\lambda}: \bmod \mathcal{C} \rightarrow \bmod A\left(\operatorname{or} F_{\lambda}^{\prime}: \bmod \mathcal{C}^{\prime} \rightarrow \bmod B\right)$ is equal to $-\underset{\mathcal{C}}{\otimes} A$ (or to $-\underset{\mathcal{C}^{\prime}}{\otimes} B$, respectively). Since these two functors are exact and map projective modules to projective modules and since the horizontal arrows of the diagram are triangle equivalences (see 4.8) we deduce that:

(1) The composition $\mathcal{D}^{b}\left(\bmod \mathcal{C}^{\prime}\right) \stackrel{F_{\lambda}^{\prime}}{\longrightarrow} \mathcal{D}^{b}(B) \stackrel{-\stackrel{\mathbb{Q}}{\otimes} T}{\longrightarrow} \mathcal{D}^{b}(\bmod A)$ is isomorphic to $-{\underset{\mathcal{C}}{\prime}}_{\mathbb{R}}^{\mathbb{L}}(B \underset{B}{\otimes} T)$.

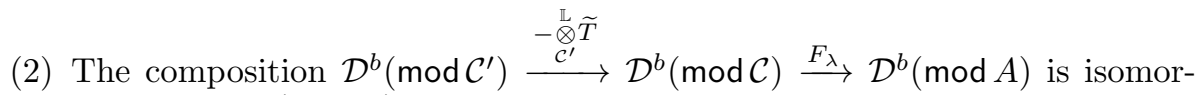
phic to $-\underset{\mathcal{C}^{\prime}}{\mathbb{L}}(\widetilde{T} \underset{\mathcal{C}}{\otimes} A)$.

On the other hand, the isomorphisms $\mu_{i}: F_{\lambda} \widetilde{T}_{i} \stackrel{\sim}{\rightarrow} T_{i}$ (for $i \in\{1, \ldots, n\}$ ) define an isomorphism $B \underset{B}{\otimes} T \stackrel{\sim}{\rightarrow} \widetilde{T} \underset{\mathcal{C}}{\otimes} A$ of $\mathcal{C}^{\prime}-A$-bimodules. This proves that the diagram commutes up to an isomorphism of functors.

Since $-{\underset{B}{\mathbb{L}}}_{B}^{\mathbb{L}} T$ is an equivalence there exists an isomorphism $\varphi_{x}: X_{x} \underset{B}{\stackrel{\mathbb{L}}{\otimes}} T \rightarrow$ $A(-, x)$ in $\mathcal{D}^{b}(\bmod A)$ with $X_{x} \in \mathcal{D}^{b}(\bmod B)$ for every $x \in \mathrm{ob}(A)$. In particular, $\bigoplus_{x \in \mathrm{ob}(A)} X_{x}$ is an indecomposable decomposition of a tilting complex in $\mathcal{D}^{b}(\bmod B)$. Then by the preceding section there exists an isomorphism $\nu_{x}: F_{\lambda}^{\prime}$ $\widetilde{X}_{x} \stackrel{\sim}{\rightarrow} X_{x}$ in $\mathcal{D}^{b}(\bmod B)$ with $\widetilde{X}_{x} \in \mathcal{D}^{b}\left(\bmod \mathcal{C}^{\prime}\right)$ for every $x \in \operatorname{ob}(A)$. By 4.8, the datum $\left(\nu_{x}\right)_{x \in \mathrm{ob}(A)}$ defines a connected Galois covering with group $G$ :

$$
\begin{aligned}
F_{\widetilde{X}, \nu}^{\prime}: \quad \operatorname{End}_{\mathcal{D}^{b}\left(\bmod \mathcal{C}^{\prime}\right)}(\widetilde{X}) & \rightarrow \operatorname{End}_{\mathcal{D}^{b}(\bmod B)}(X), \\
{ }^{g} \widetilde{X}_{x} & \mapsto X_{x}, \\
{ }^{g} \widetilde{X}_{x} \stackrel{u}{\rightarrow}{ }^{h} \widetilde{X}_{y} & \mapsto X_{x} \stackrel{\nu_{y} F_{\lambda}^{\prime}(u) \nu_{x}^{-1}}{\rightarrow} X_{y} .
\end{aligned}
$$

On the other hand, the isomorphisms $\varphi_{x}$ (for $x \in \mathrm{ob}(A)$ ) define the following isomorphism of $k$-categories:

$$
\begin{aligned}
\rho_{X, \varphi}: \quad \operatorname{End}_{\mathcal{D}^{b}(\bmod B)}(X) & \rightarrow A, \\
X_{x} & \mapsto x, \\
X_{x} \stackrel{u}{\rightarrow} X_{y} & \mapsto\left(\varphi_{y} \circ(u \otimes T) \circ \varphi_{x}^{-1}\right)\left(\operatorname{Id}_{x}\right) \in A(x, y) .
\end{aligned}
$$


Thus, we have a connected Galois covering $\rho_{X, \varphi} \circ F_{\widetilde{X}, \nu}^{\prime}: \operatorname{End}_{\mathcal{D}^{b}\left(\bmod \mathcal{C}^{\prime}\right)}(\widetilde{X}) \rightarrow A$ with group $G$ which we denote by $F^{\prime \prime}$. The following lemma relates $F$ and $F^{\prime \prime}$.

Lemma 5.3. The Galois coverings $F$ and $F^{\prime \prime}$ are equivalent.

Proof. We need to construct a commutative diagram:

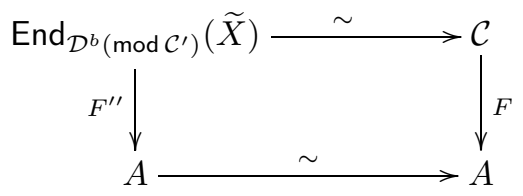

where the horizontal arrows are isomorphisms and the bottom horizontal isomorphism extends the identity map on objects. For this purpose, we proceed in two steps.

Step 1: We express $F$ as a functor between subcategories of $\mathcal{D}^{b}(\bmod \mathcal{C})$ and $\mathcal{D}^{b}(\bmod A)$. Given $x \in \operatorname{ob}(\mathcal{C})$, the $A$-module $F_{\lambda}(\mathcal{C}(-, x))$ depends only on $F(x)$ (and not on $x$ ) because $F_{\lambda}$ is $G$-invariant. Besides, there is a canonical isomorphism $\iota_{x}: F_{\lambda}(\mathcal{C}(-, x)) \stackrel{\sim}{\rightarrow} A(-, F(x))$ of $A$-modules induced by $F:$ If $y \in \mathrm{ob}(A)$, then $\left(F_{\lambda}(\mathcal{C}(-, x))\right)(y)=\bigoplus_{F y^{\prime}=F y} \mathcal{C}\left(y^{\prime}, x\right)$ and an element $\left(u_{y^{\prime}}\right)_{y^{\prime}}$ of this vector space is mapped by $\iota_{x}$ to $\sum_{y^{\prime}} F\left(u_{y^{\prime}}\right) \in A(F(y), F(x))$. Clearly, this isomorphism depends only on $F(x)$ (and not on $x$ ). Now let $\mathcal{P}_{A}$ and $\mathcal{P}_{\mathcal{C}}$ be the full subcategories of $\mathcal{D}^{b}(\bmod A)$ and $\mathcal{D}^{b}(\bmod \mathcal{C})$ with object sets $\{A(-, x) \mid x \in \operatorname{ob}(A)\}$ and $\{\mathcal{C}(-, x) \mid x \in$ $\mathrm{ob}(\mathcal{C})\}$, respectively. We have a commutative diagram:

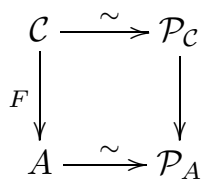

where the unlabelled functors are as follows:

(1) The functor $\mathcal{C} \rightarrow \mathcal{P}_{\mathcal{C}}$ is the following isomorphism:

$$
\begin{aligned}
\mathcal{C} & \rightarrow \mathcal{P}_{\mathcal{C}}, \\
x \in \mathrm{ob}(\mathcal{C}) & \mapsto \mathcal{C}(-, x), \\
u \in \mathcal{C}(x, y) & \mapsto \mathcal{C}(-, u): \mathcal{C}(-, x) \rightarrow \mathcal{C}(-, y) .
\end{aligned}
$$

(2) The functor $A \rightarrow \mathcal{P}_{A}$ is the following isomorphism:

$$
\begin{aligned}
A & \rightarrow \mathcal{P}_{A}, \\
x \in \mathrm{ob}(A) & \mapsto A(-, x), \\
u \in A(x, y) & \mapsto A(-, u): A(-, x) \rightarrow A(-, y) .
\end{aligned}
$$

(3) The functor $\mathcal{P}_{\mathcal{C}} \rightarrow \mathcal{P}_{A}$ is as follows:

$$
\begin{aligned}
\mathcal{P}_{\mathcal{C}} & \rightarrow \mathcal{P}_{A}, \\
\mathcal{C}(-, x) & \mapsto A(-, F(x)), \\
\mathcal{C}(-, x) \stackrel{u}{\rightarrow} \mathcal{C}(-, y) & \mapsto A(-, F(x)) \stackrel{\iota_{y} \circ F_{\lambda}(u) \circ \iota_{x}^{-1}}{\longrightarrow} A(-, F(y)) .
\end{aligned}
$$

In particular, $\mathcal{P}_{\mathcal{C}} \rightarrow \mathcal{P}_{A}$ is a Galois covering with group $G$. 
Step 2: We now relate $F^{\prime \prime}$ to the Galois covering $\mathcal{P}_{\mathcal{C}} \rightarrow \mathcal{P}_{A}$. We first construct an isomorphism $\operatorname{End}_{\mathcal{D}^{b}\left(\bmod \mathcal{C}^{\prime}\right)}(\widetilde{X}) \stackrel{\sim}{\rightarrow} \mathcal{P}_{\mathcal{C}}$. Let $\Psi: F_{\lambda}^{\prime}(-) \underset{B}{\stackrel{\mathbb{Q}}{\otimes}} T \stackrel{\sim}{\rightarrow} F_{\lambda}\left(-{\underset{\mathcal{C}}{\otimes}}_{\mathcal{Q}}^{\mathbb{L}} \widetilde{T}\right)$ be an isomorphism of functors (see 5.2). Let $x \in \mathrm{ob}(A)$. So we have a composition of isomorphisms in $\mathcal{D}^{b}(\bmod A)$ :

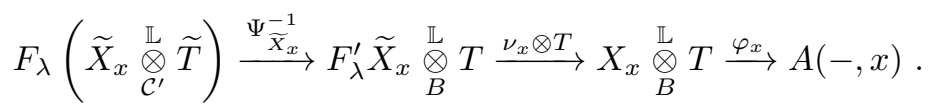

Therefore, by 2.3, there exists an isomorphism $\psi_{x}: \widetilde{X}_{x} \underset{\mathcal{C}^{\prime}}{\stackrel{\mathbb{R}}{\otimes}} \widetilde{T} \stackrel{\sim}{\longrightarrow} \mathcal{C}(-, L(x))$ in $\mathcal{D}^{b}(\bmod \mathcal{C})$ with $L(x) \in F^{-1}(x)$. We deduce that the following is an isomorphism of $k$-categories because $-\underset{\mathcal{C}^{\prime}}{\mathbb{L}} \widetilde{T}$ is a $G$-equivariant functor (see 4.8):

$$
\begin{aligned}
\operatorname{End}_{\mathcal{D}^{b}\left(\bmod \mathcal{C}^{\prime}\right)}(\widetilde{X}) & \rightarrow \mathcal{P}_{\mathcal{C}}, \\
{ }^{g} \widetilde{X}_{x} & \mapsto \mathcal{C}(-, g L(x)), \\
{ }^{g} \widetilde{X}_{x} \stackrel{{ }^{u}}{\rightarrow}{ }^{h} \widetilde{X}_{y} & \mapsto \mathcal{C}(-, g L(x)) \stackrel{{ }^{h} \psi_{y} \circ(u \otimes \widetilde{T}) \circ\left({ }^{g} \psi_{x}\right)^{-1}}{\longrightarrow} \mathcal{C}(-, h L(y)) .
\end{aligned}
$$

We now construct another isomorphism between $A$ and $\mathcal{P}_{A}$. We have the following composition of isomorphisms in $\mathcal{D}^{b}(\bmod A)$ which we denote by $\alpha_{x}$ :

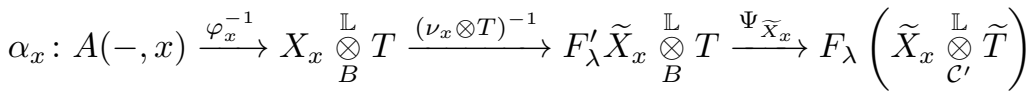

$$
\begin{aligned}
& \stackrel{F_{\lambda}\left(\psi_{x}\right)}{\longrightarrow} F_{\lambda}(\mathcal{C}(-, L(x))) \stackrel{\iota_{x}}{\longrightarrow} A(-, x) .
\end{aligned}
$$

Note that $\alpha_{x}: A(-, x) \stackrel{\sim}{\longrightarrow} A(-, x)$ is necessarily equal to the multiplication by a scalar in $k^{*}$ because $A(-, x)$ is an indecomposable projective $A$-module and $A$ is piecewise hereditary. Therefore, we have an isomorphism of categories:

$$
\begin{aligned}
A & \rightarrow \mathcal{P}_{A}, \\
x & \mapsto A(-, x), \\
u \in A(x, y) & \mapsto \alpha_{y} \circ A(-, u) \circ \alpha_{x}^{-1} .
\end{aligned}
$$

Hence the horizontal arrows of the following diagram are isomorphisms:

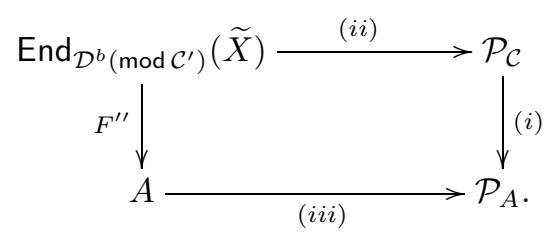

We claim that this diagram commutes. The commutativity is clearly satisfied on objects. Let $u:{ }^{g} \widetilde{X}_{x} \rightarrow{ }^{h} \widetilde{X}_{y}$ be a morphism in $\operatorname{End}_{\mathcal{D}^{b}\left(\bmod \mathcal{C}^{\prime}\right)}(\widetilde{X})$. Denote by 
$u_{1}: A(-, x) \rightarrow A(-, y)$ the image of $u$ under the composition of $(i)$ and $(i i)$. Then:

$$
\begin{aligned}
& u_{1}=\iota_{y} \circ F_{\lambda}\left({ }^{h} \psi_{y} \circ(u \otimes \widetilde{T}) \circ\left({ }^{g} \psi_{x}\right)^{-1}\right) \circ \iota_{x}^{-1} \\
& =\iota_{y} \circ F_{\lambda}\left(\psi_{y}\right) \circ F_{\lambda}(u \otimes \widetilde{T}) \circ\left(F_{\lambda}\left(\psi_{x}\right)\right)^{-1} \circ \iota_{x}^{-1} \\
& \text { because } F_{\lambda} \text { is } G \text {-invariant, } \\
& u_{1}=\iota_{y} \circ F_{\lambda}\left(\psi_{y}\right) \circ \Psi_{\tilde{X}_{y}} \circ\left(F_{\lambda}^{\prime}(u) \otimes T\right) \circ \Psi_{\tilde{X}_{x}}^{-1} \circ\left(F_{\lambda}\left(\psi_{x}\right)\right)^{-1} \circ \iota_{x}^{-1} \\
& \text { by definition of } \Psi \text {, } \\
& u_{1}=\alpha_{y} \circ \varphi_{y} \circ\left(\nu_{y} \otimes T\right) \circ\left(F_{\lambda}^{\prime}(u) \otimes T\right) \circ\left(\nu_{x} \otimes T\right)^{-1} \circ \varphi_{x}^{-1} \circ \alpha_{x}^{-1} \\
& \text { by definition of } \alpha_{x} \text { and } \alpha_{y} \text {, } \\
& =\alpha_{y} \circ \varphi_{y} \circ\left(\left(\nu_{y} \circ F_{\lambda}^{\prime}(u) \circ \nu_{x}^{-1}\right) \otimes T\right) \circ \varphi_{x}^{-1} \circ \alpha_{x}^{-1} \text {. }
\end{aligned}
$$

Now, let $u_{2} \in A(x, y)$ be the image of $u$ under $F^{\prime \prime}$, that is,

$$
u_{2}=\left(\varphi_{y} \circ\left(\left(\nu_{y} \circ F_{\lambda}^{\prime}(u) \circ \nu_{x}^{-1}\right) \otimes T\right) \circ \varphi_{x}^{-1}\right)\left(\operatorname{Id}_{x}\right) .
$$

Therefore, $A\left(-, u_{2}\right)$ is equal to the morphism $\varphi_{y} \circ\left(\left(\nu_{y} \circ F_{\lambda}^{\prime}(u) \circ \nu_{x}^{-1}\right) \otimes T\right) \circ \varphi_{x}^{-1}$ : $A(-, x) \rightarrow A(-, y)$ of $\mathcal{P}_{A}$. In particular, the image of $u_{2}$ under (iii) coincides with $u_{1}$. Therefore, $\left(D_{2}\right)$ is commutative. Since $\left(D_{1}\right)$ also commutes, we deduce that $(D)$ does as well. Thus, $F$ and $F^{\prime \prime}$ are equivalent.

\section{Correspondence between the Galois coverings of $A$ and those of $B$.}

Proposition 5.4. Let $A$ be an algebra derived equivalent to a hereditary abelian category $\mathcal{H}$ such that $\overrightarrow{\mathcal{K}}_{\mathcal{H}}$ is connected. Let $T \in \mathcal{D}^{b}(\bmod A)$ be a tilting complex, $B=\operatorname{End}_{\mathcal{D}^{b}(\bmod A)}(T)$ and $G$ a group. With the notation of [5.1, the map $[F] \mapsto[F]_{T}$ is a well-defined bijection from the set of equivalence classes of connected Galois coverings with group $G$ of $A$ to the set of equivalence classes of Galois coverings with group $G$ of $B$.

Proof. Let $\mathrm{Gal}_{A}(G)$ be the set of equivalence classes of connected Galois coverings with group $G$ of $A$. By 5.1, there is a well-defined map:

$$
\begin{aligned}
\gamma_{A}: \operatorname{Gal}_{A}(G) & \rightarrow \operatorname{Gal}_{B}(G), \\
{[F] } & \mapsto[F]_{T} .
\end{aligned}
$$

We keep the notation $X_{x}, \varphi_{x}$ (for $x \in \mathrm{ob}(A)$ ) introduced after the proof of 5.2 . Then we also have a well-defined map:

$$
\begin{aligned}
& \gamma_{B}: \operatorname{Gal}_{B}(G) \rightarrow \operatorname{Gal}_{\text {End }_{\mathcal{D}^{b}(\bmod B)}(X)}(G), \\
& {[F] \mapsto[F]_{X} .}
\end{aligned}
$$

By 5.3 we know that $\gamma_{A}$ is injective and $\gamma_{B}$ is surjective. Therefore, $\gamma_{A}$ is bijective because $A, T$ and $B, X$ play symmetric roles.

By 5.4 we have some information on the existence of a universal cover. Indeed, we have the following result.

Proposition 5.5. Let $A$ be as in 5.4 and let $T \in \mathcal{D}^{b}(\bmod A)$ a tilting complex. Assume that $A$ admits a universal cover $\widetilde{F}: \widetilde{\mathcal{C}} \rightarrow A$. Then $\operatorname{End}_{\mathcal{D}^{b}(\bmod A)}(T)$ admits a universal cover with group isomorphic to the one of $\widetilde{F}$. 
Proof. Fix an indecomposable decomposition $T=T_{1} \oplus \cdots \oplus T_{n}$. Let $B=$ $\operatorname{End}_{\mathcal{D}^{b}(\bmod A)}(T)$. So $B$ is the full subcategory of $\mathcal{D}^{b}(\bmod A)$ with objects $T_{1}, \ldots, T_{n}$. Let $x_{0} \in \mathrm{ob}(A)$ be a basepoint for the category $\operatorname{Gal}\left(A, x_{0}\right)$ of pointed Galois coverings of $A$. We construct a (full and faithful) functor $\widetilde{F} \rightarrow \rightarrow \operatorname{Gal}\left(B, T_{1}\right)$. Recall that $\widetilde{F}^{\rightarrow}$ was defined in Section 1 and there is at most one morphism between two pointed Galois coverings. We need the following data:

(1) For every $i \in\{1, \ldots, n\}$, let $\widetilde{T}_{i} \in \bmod \widetilde{\mathcal{C}}$ be such that $\widetilde{F}_{\lambda} \widetilde{T}_{i} \simeq T_{i}$. Therefore the $k$-categories $B=\operatorname{End}_{\mathcal{D}^{b}(\bmod A)}(T)$ and $\operatorname{End}_{\mathcal{D}^{b}(\bmod A)}\left(\bigoplus_{i=1}^{n} F_{\lambda} \widetilde{T}_{i}\right)$ are isomorphic. For simplicity, we assume that $\widetilde{F}_{\lambda} \widetilde{T}_{i}=T_{i}$ for every $i$.

(2) If $F \in \widetilde{F}^{\rightarrow}$, there exists a unique morphism $p: \widetilde{F} \rightarrow F$ in $\operatorname{Gal}\left(A, x_{0}\right)$. Since $p$ is a Galois covering ([32, Prop. 3.4]), we set $T_{i}^{F}=p_{\lambda} \widetilde{T}_{i}$ for every $i$. Then:

(i) We have $T_{i}=F_{\lambda}\left(T_{i}^{F}\right)$ for every $i \in\{1, \ldots, n\}$ and $F \in \widetilde{F}^{\rightarrow}$. Indeed, there exists a unique morphism $p: \widetilde{F} \rightarrow F$, so that $\widetilde{F}_{\lambda}=F_{\lambda} \circ p_{\lambda}$.

(ii) Let $u: F \rightarrow F^{\prime}$ be a morphism in $\widetilde{F}^{\rightarrow}$. Let $G$ be the group of $F$ and $G^{\prime}$ the group of $F^{\prime}$. Then $u$ is a Galois covering (32, Prop. 3.4]). Let $p: \widetilde{F} \rightarrow F$ and $p^{\prime}: \widetilde{F} \rightarrow F^{\prime}$ be the unique morphisms in $\operatorname{Gal}\left(A, x_{0}\right)$ from $\widetilde{F}$ to $F$ and from $\widetilde{F}$ to $F^{\prime}$, respectively. Then $p^{\prime}=u \circ p$ because of [32, Lem. 3.1] and because we are dealing with pointed Galois coverings. Therefore, $u_{\lambda}\left(T_{i}^{F}\right)=$ ${ }^{\sigma_{u}(g)} T_{i}^{F^{\prime}}$ for every $i \in\{1, \ldots, n\}$ and every $g \in G$. Here, $\sigma_{u}: G \rightarrow G^{\prime}$ is the unique (surjective) group homomorphism such that $u \circ g=\sigma_{u}(g) \circ u$ for every $g \in G$ ([32, Prop. 3.4]).

Now we can construct a functor $\widetilde{F}^{\rightarrow} \rightarrow \operatorname{Gal}\left(B, T_{1}\right)$. Let $F:(\mathcal{C}, x) \rightarrow\left(A, x_{0}\right)$ be in $\widetilde{F}^{\rightarrow}$. Let $G$ be the group of $F$. By $(i)$ and 4.8, we have a pointed Galois covering with group $G$ induced by $F_{\lambda}: \mathcal{D}^{b}(\bmod \mathcal{C}) \rightarrow \mathcal{D}^{b}(\bmod A)$ :

$$
\begin{aligned}
F_{T}:\left(\operatorname{End}_{\mathcal{D}^{b}(\bmod \mathcal{C})}\left(\bigoplus_{g, i}{ }^{g} T_{i}^{F}\right), T_{1}^{F}\right) & \rightarrow\left(B, T_{1}\right), \\
{ }^{g} T_{i}^{F} & \mapsto T_{i}, \\
{ }^{g} T_{i}^{F} \stackrel{f}{\rightarrow}{ }^{h} T_{j}^{F} & \mapsto T_{i} \stackrel{F_{\lambda}(f)}{\longrightarrow} T_{j} .
\end{aligned}
$$

So $\left[F_{T}\right]=[F]_{T}$. Thus, we have associated a pointed Galois covering with group $G$ of $B$ to any pointed Galois covering with group $G$ of $A$. Now we associate a morphism of pointed Galois coverings of $B$ to any morphism of pointed Galois coverings of $A$. Let $u: F \rightarrow F^{\prime}$ be a morphism in $\widetilde{F}^{\rightarrow}$ where $F:(\mathcal{C}, x) \rightarrow\left(A, x_{0}\right)$ and $F^{\prime}:\left(\mathcal{C}^{\prime}, x^{\prime}\right) \rightarrow\left(A, x_{0}\right)$ have groups $G$ and $G^{\prime}$, respectively. By $(i i)$, we have a well-defined $k$-linear pointed functor induced by $u_{\lambda}: \mathcal{D}^{b}(\bmod \mathcal{C}) \rightarrow \mathcal{D}^{b}\left(\bmod \mathcal{C}^{\prime}\right)$ :

$$
\begin{aligned}
u_{T}:\left(\operatorname{End}_{\mathcal{D}^{b}(\bmod \mathcal{C})}\left(\bigoplus_{g, i}{ }^{g} T_{i}^{F}\right), T_{1}^{F}\right) & \rightarrow\left(\operatorname{End}_{\mathcal{D}^{b}\left(\bmod \mathcal{C}^{\prime}\right)}\left(\bigoplus_{g^{\prime}, i}{ }^{g^{\prime}} T_{i}^{F^{\prime}}\right), T_{1}^{F^{\prime}}\right), \\
{ }^{g} T_{i}^{F} & \mapsto u_{\lambda}\left({ }^{g} T_{i}^{F}\right)={ }^{\sigma_{u}(g)} T_{i}^{F^{\prime}} \\
{ }^{g} T_{i}^{F} \stackrel{f}{\rightarrow}{ }^{h} T_{j}^{F} & \mapsto{ }^{\sigma_{u}(g)} T_{i}^{F^{\prime}} \stackrel{u_{\lambda}(f)}{\longrightarrow}{ }^{\sigma_{u}(h)} T_{j}^{F^{\prime}} .
\end{aligned}
$$


The equality $u_{\lambda}\left({ }^{g} T_{i}^{F}\right)={ }^{\sigma_{u}(g)} T_{i}^{F^{\prime}}$ follows from the equality $u \circ g=\sigma_{u}(g) \circ u$. Also, $u_{\lambda}\left(T_{1}^{F}\right)=T_{1}^{F^{\prime}}$. Since $F^{\prime} \circ u=F$ and $F_{T}, F_{T}^{\prime}$ and $u_{T}$ are defined as restrictions of $F_{\lambda}$, $F_{\lambda}^{\prime}$ and $u_{\lambda}$, respectively, we infer that $u_{T}: F_{T} \rightarrow F_{T}^{\prime}$ is a morphism in $\operatorname{Gal}\left(B, T_{1}\right)$. Thus, to any morphism in $\widetilde{F}^{\rightarrow}$ we have associated a morphism in $\operatorname{Gal}\left(B, T_{1}\right)$. We let the reader check that the following is a functor:

$$
\begin{aligned}
\Psi: \widetilde{F}^{\rightarrow} & \rightarrow \operatorname{Gal}\left(B, T_{1}\right), \\
F & \mapsto F_{T}, \\
F \stackrel{u}{\rightarrow} F^{\prime} & \mapsto F_{T} \stackrel{u_{T}}{\longrightarrow} F_{T}^{\prime} .
\end{aligned}
$$

Also, it is not difficult to prove that $\Psi$ is full and faithful, although we shall not use this fact in the sequel. Note that the Galois covering $F_{T}$ lies in $\Psi(\widetilde{F}) \rightarrow$ for every $F \in \widetilde{F}^{\rightarrow}$.

Now we can prove that $\Psi(\widetilde{F})$ is a universal cover for $B$. Let $F$ be a connected Galois covering of $B$. By [5.4 there exists a connected Galois covering $F^{\prime}$ of $A$ such that $[F]=\left[F^{\prime}\right]_{T}$. Since $\widetilde{F}$ is a universal cover of $A$, the Galois covering $F^{\prime}$ of $A$ is equivalent to some $F^{\prime \prime} \in \widetilde{F}^{\rightarrow}$, that is, $\left[F^{\prime}\right]=\left[F^{\prime \prime}\right]$. As noticed above, we have $\left[F_{T}^{\prime \prime}\right]=\left[F^{\prime \prime}\right]_{T}$. Therefore, $[F]=\left[F^{\prime}\right]_{T}=\left[F^{\prime \prime}\right]_{T}=\left[F_{T}^{\prime \prime}\right]$, that is, $F$ is equivalent to a Galois covering of $B$ lying in $\Psi(\widetilde{F}) \rightarrow$. So $\Psi(\widetilde{F})$ is a universal Galois covering of $B$.

\section{The MAIN TheOREM AND ITS COROLlary}

In this section, we prove Theorem $\mathrm{A}$ and Corollary $\mathrm{B}$ We assume that $A$ is a connected algebra derived equivalent to a hereditary abelian category $\mathcal{H}$ such that $\overrightarrow{\mathcal{K}}_{\mathcal{H}}$ is connected. We refer the reader to [11, [20] for the computation of the Hochschild cohomology of hereditary algebras and to [21] for that of piecewise hereditary algebras.

Two particular cases: Paths algebras and squid algebras. We first check that our main results hold true for paths algebras and for squid algebras.

Lemma 6.1. Assume that $A=k Q$ where $Q$ is a finite connected quiver with no oriented cycles. Then Theorem $\mathrm{A}$ and Corollary $\mathrm{B}$ hold true for A.

Proof. Let $\widetilde{Q} \rightarrow Q$ be the universal Galois covering of quivers (see [38]). It follows from [32, Prop. 4.4] that the induced Galois covering $k \widetilde{Q} \rightarrow k Q$ with group $\pi_{1}(Q)$ is a universal cover of $A$. Whence Theorem $\AA$ for $A=k Q$. On the other hand, $\mathrm{HH}^{1}(k Q)=0$ if and only if $Q$ is a tree $([11,[20,1.6])$. Whence Corollary B,

We now turn to the case of squid algebras. We refer the reader to 42 for more details on squid algebras. A squid algebra over an algebraically closed field $k$ is defined by the following data: An integer $t \geqslant 2$, a sequence $p=\left(p_{1}, \ldots, p_{t}\right)$ of nonnegative integers and a sequence $\tau=\left(\tau_{3}, \ldots, \tau_{t}\right)$ of pairwise distinct nonzero elements of $k$. With this data, the squid algebra $S(t, p, \tau)$ is the $k$-algebra $k Q / I$ where $Q$ is the following quiver:

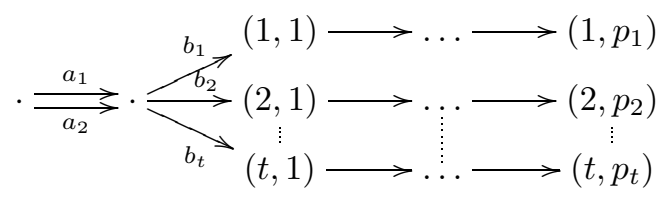


and $I$ is the ideal generated by the following relations: $b_{1} a_{1}=b_{2} a_{2}=0, b_{i} a_{2}=$ $\tau_{i} b_{i} a_{1}$ for $i=3, \ldots, t$.

Using Happel's long exact sequence ([20]), one can compute $\mathrm{HH}^{1}(S(t, p, \tau))$ :

$$
\operatorname{dim}_{k} \mathrm{HH}^{1}(S(t, p, \tau))= \begin{cases}1 & \text { if } t=2 \\ 0 & \text { if } t \geqslant 3\end{cases}
$$

Following $[38$ the bound quiver $(Q, I)$ defines a Galois covering $k \widetilde{Q} / \widetilde{I} \rightarrow k Q / I$ with group isomorphic to $\mathbb{Z}$ if $t=2$ and with trivial group otherwise. One can easily check that this Galois covering is universal in the sense of Theorem A. The above considerations give the following.

Lemma 6.2. Let $A$ be a squid algebra. Then Theorem $\mathrm{A}$ and Corollary $\mathrm{B}$ hold true for $A$.

The general case. Using $5.5,6.1$ and 6.2 we can prove the two main results of this text.

Proof of Theorem $\mathrm{A}$ and Corollary $\mathrm{B}$. Assume first that $A$ is piecewise hereditary of type $Q$ where $Q$ is a finite connected quiver with no oriented cycles. So there exists a tilting complex $T \in \mathcal{D}^{b}(\bmod k Q)$ such that $A \simeq \operatorname{End}_{\mathcal{D}^{b}(\bmod k Q)}(T)$. By 5.5 and 6.1 the algebra $A$ admits a universal Galois covering with group isomorphic to the fundamental group of $Q$. In particular, $A$ is simply connected if and only if $Q$ is a tree. On the other hand, $Q$ is a tree if and only if $\mathrm{HH}^{1}(k Q)=0$ ([11, 20, 1.6]) and $\mathrm{HH}^{1}(k Q) \simeq \mathrm{HH}^{1}(A)([30])$. Therefore, $A$ is simply connected if and only if $\mathrm{HH}^{1}(A)=0$, that is, if and only if $Q$ is a tree.

Now assume that $A$ is not derived equivalent to $\mathcal{D}^{b}(\bmod k Q)$ for any finite quiver Q. Then [24, Prop. 2.1, Thm. 2.6] implies that there exists a squid algebra $S=S(t, p, \tau)$ and a tilting complex $T \in \mathcal{D}^{b}(\bmod S)$ such that $A \simeq \operatorname{End}_{\mathcal{D}^{b}(\bmod S)}(T)$. By 5.5 and 6.2, the algebra $A$ has a universal cover with group isomorphic to the trivial group or to $\mathbb{Z}$ according to whether $t \geqslant 3$ or $t=2$. In particular, $A$ is simply connected if and only if $t \geqslant 3$, that is, if and only if $\mathrm{HH}^{1}(S)=0$ (6.2). Since $\mathrm{HH}^{1}(S) \simeq \mathrm{HH}^{1}(A)([30])$, we deduce that $A$ is simply connected if and only if $\mathrm{HH}^{1}(A)=0$.

\section{ACKNOWLEDGEMENTS}

The author thanks Ibrahim Assem for his encouragements. Also, he would like to thank an anonymous referee for pointing out many references to which the present work is related.

\section{REFERENCES}

1. I. Assem, E. N. Marcos, and J. A. de La Peña, The simple connectedness of a tame tilted algebra, J. Algebra 237 (2001), no. 2, 647-656. MR.1816708 (2002b:16025)

2. I. Assem, D. Simson, and A. Skowroński, Elements of the representation theory of associative algebras. Vol. 1: Techniques of representation theory., London Mathematical Society Student Texts 65. Cambridge: Cambridge University Press. ix, 458 pp. , 2006. MR2197389 (2006j:16020)

3. I. Assem and A. Skowroński, Iterated tilted algebras of type $\tilde{\mathbb{A}}_{n}$, Math. Z. 195 (1987), 269-290. MR892057 (88m:16033)

4. , Algebras with cycle-finite derived categories, Math. Ann 280 (1988), 441-463. MR936322 (89f:16023b) 
5. - On some classes of simply connected algebras, Proc. London Math. Soc. 56 (1988), no. 3, 417-450. MR931509 (89f:16023a)

6. __ On tame repetitive algebras, Fund. Math. 142 (1993), 59-84. MR.1207471 (94a:16022)

7. K. Bongartz and P. Gabriel, Covering spaces in representation theory, Invent. Math. 65 (1982), 331-378. MR643558 (84i:16030)

8. A. B. Buan, R. Marsh, M. Reineke, I. Reiten, and G. Todorov, Tilting theory and cluster combinatorics, Adv. Math. 204 (2006), no. 2, 572-618. MR2249625 (2007f:16033)

9. R.-O. Buchweitz and S. Liu, Hochschild cohomology and representation-finite algebras., Proc. London Math. Soc., Ser. III 88 (2004), no. 2, 355-380. MR2032511 (2004m:16010)

10. P. Caldero, F. Chapoton, and R. Schiffler, Quivers with relations arising from clusters $\left(A_{n}\right.$ case), Trans. Amer. Math. Soc. 358 (2006), no. 3, 1347-1364. MR2187656 (2007a:16025)

11. C. Cibils, On the Hochschild cohomology of finite dimensional algebras., Comm. Algebra 16 (1988), no. 3, 645-649. MR917337 (89c:16027)

12. C. Cibils and E. N. Marcos, Skew categories, Galois coverings and smash-product of a $k$ category, Proc. Amer. Math. Soc. 134 (2006), no. 1, 39-50. MR2170541(2006e:18003)

13. J. Dionne, M. Lanzilotta, and D. Smith, Skew group algebras of piecewise hereditary algebras are piecewise hereditary, J. Pure Appl. Algebra 213 (2009), no. 2, 241-249. MR2467401

14. P. Dowbor and A. Skowroński, Galois coverings of representation infinite algebras, Comment. Math. Helv. 62 (1987), 311-337. MR896100 (88m:16020)

15. K. Erdmann, O. Kerner, and A. Skowroński, Self-injective algebras of wild tilted type, J. Pure Appl. Algebra 149 (2000), 127-176. MR1757729 (2001g:16033)

16. S. Fomin and A. Zelevinsky, Cluster algebras I: Foundations, Trans. Amer. Math. Soc. 353 (2002), 497-529. MR1887642 (2003f:16050)

17. P. Gabriel, The universal cover of a representation-finite algebra, Representations of algebras: Proceedings of the third International Conference on Representations of Algebras, Puebla/Mexico 1980, Lecture Notes in Math., vol. 903, Springer-Verlag, 1981, pp. 68-105. MR654725 (83f:16036)

18. W. Geigle and H. Lenzing, A class of weighted projective curves arising in representation theory of finite-dimensional algebras, Singularities, representations of algebras, and vector bundles (Lambrecht, 1985), Lecture Notes in Mathematics, vol. 1273, Springer-Verlag, Berlin, 1987, pp. 265-297. MR915180 (89b:14049)

19. D. Happel, Triangulated categories in the representation theory of finite dimensional algebras, London Mathematical Society Lecture Notes Series, vol. 119, Cambridge University Press, Cambridge, 1988. MR 935124 (89e:16035)

20. _ Hochschild cohomology of finite dimensional algebras, Séminaire d'Algèbre Paul Dubreuil, Marie-Paule Malliavin, Lecture Notes in Mathematics 1404 (1989), 108-126. MR 1035222 (91b:16012)

21. Hochschild cohomology of piecewise hereditary algebras, Colloq. Math. 78 (1998), no. 2, 261-266. MR.1659132 (99j:16002)

22. _ A characterisation of hereditary categories with tilting object, Invent. Math. 144 (2001), 381-398. MR1827736 (2002a:18014)

23. D. Happel and I. Reiten, Hereditary categories with tilting object, Math. Z. 232 (1999), 559588. MR 1719670 (2001d:18012)

24. _ Hereditary abelian categories with tilting object over arbitrary base fields., J. Algebra 256 (2002), no. 2, 414-432. MR1939113 (2004b:16016)

25. D. Happel, I. Reiten, and S. O. Smalø, Piecewise hereditary algebras, Arch. Math. 66 (1996), no. 3, 182-186. MR.1373243 (96m:16019)

26. D. Happel, J. Rickard, and A. Schofield, Piecewise hereditary algebras, Bull. London Math. Soc. 20 (1988), no. 1, 23-28. MR.916069 (89c:16039)

27. D. Happel and L. Unger, On the set of tilting objects in hereditary categories, Representations of algebras and related topics, Fields Inst. Commun., vol. 45, Amer. Math. Soc., 2005, pp. 141159. MR2146246 (2006h:18006)

28. D. Happel and D. Zacharia, A homological characterisation of piecewise hereditary algebras, Math. Z. 260 (2008), no. 1, 177-185. MR2413349 (2009g:16011)

29. D. Hugues and J. Waschbãsch, Trivial extensions of tilted algebras, Proc. London Math. Soc. 46 (1983), 347-364. MR693045 (84m:16023)

30. B. Keller, Hochschild cohomology and derived Picard groups, J. Pure Appl. Algebra 190 (2004), 177-196. MR2043327 (2004m:16012) 
31. O. Kerner, A. Skowroński, and K. Yamagata, Invariance of selfinjective algebras of quasitilted type under derived equivalences, Manuscripta Math. 119 (2006), 359-381. MR 2207856 (2007b:16008)

32. P. Le Meur, The universal cover of an algebra without double bypass., J. Algebra 312 (2007), no. 1, 330-353. MR2320460 (2008c:16026)

33. _ On Galois coverings and tilting modules, J. Algebra 319 (2008), no. 12, 4961-4999. MR2423814 (2009e:16026)

34. The universal cover of a monomial algebra without multiple arrows, J. Algebra Appl. 7 (2008), no. 4, 443-469. MR.2442071

35. H. Lenzing and A. Skowroński, Quasi-tilted algebras of canonical type, Colloq. Math. 71 (1996), no. 2, 161-181. MR1414820 (97j:16019)

36. , Derived equivalence as iterated tilting, Arch. Math. 76 (2001), no. 1, 20-24. MR:1808737 (2001m:16015)

37. _ Selfinjective algebras of wild canonical type, Colloq. Math. 96 (2003), 245-275. MR2010359 (2004h:16013)

38. R. Martínez-Villa and J. A. de la Peña, The universal cover of a quiver with relations, J. Pure Appl. Algebra 30 (1983), 277-292. MR724038 (85f:16035)

39. J. Rickard, Morita theory for derived categories, J. London Math. Soc. (1989), no. 39, 43-456. MR:1002456 (91b:18012)

40. $ـ$ Derived equivalences as derived functors, J. London Math. Soc. 43 (1991), 37-48. MR:1099084 (92b:16043)

41. C. Riedtmann, Algebren, Darstellungsköcher, Ueberlagerungen und zurück, Comm. Math. Helv. 55 (1980), 199-224. MR576602 (82k:16039)

42. C. M. Ringel, The canonical algebras (with an appendix by W. Crawley-Boevey), Banach Center Publications 26 (1990), 407-432. MR.1171247 (93e:16022)

43. D. Simson and A. Skowroński, Elements of the representation theory of associative algebras, Vol. 2, London Mathematical Society Student Texts, vol. 71, Cambridge University Press, 2007. MR2360503 (2009f:16001)

44. __ Elements of the representation theory of associative algebras, Vol. 3, London Mathematical Society Student Texts, vol. 72, Cambridge University Press, 2007. MR2382332 (2008m:16001)

45. A. Skowroński, On algebras with finite strong global dimension, Bull. Polish. Acad. Sci. Math. 35 (1987), no. 9-10, 539-547. MR937729 (89b:16019)

46. Selfinjective algebras of polynomial growth, Math. Ann. 285 (1989), 177-199. MR.1016089 (90k:16024)

47. - Algebras of polynomial growth, Topics in algebra, Part 1, vol. 26, Banach Center Publ., no. 1, PWN, Warsaw, 1990, pp. 535-568. MR.1171252 (93k:16026)

48. A. Skowroński, Simply connected algebras and Hochschild cohomologies, Can. Math. Soc. Conf. Proc. 14 (1993), 431-447. MR.1265301

49. A. Skowroński, Selfinjective algebras: finite and tame type, Trends in Representation Theory and Related Topics, Contem. Math., vol. 406, Amer. Math. Soc., 2006, pp. 168-238. MR 2258046 (2007f:16045)

50. A. Skowroński and K. Yamagata, Stable equivalence of selfinjective algebras of tilted type, Arch. Math. 70 (1998), 341-350. MR.1612578 (99g:16017)

51. _ On invariability of self-injective algebras of tilted type under stable equivalences, Proc. Amer. Math. Soc. 132 (2003), no. 3, 659-667. MR2019940 (2005c:16031)

52. Stable equivalence of selfinjective algebras of Dynkin type, Algebr. Represent. Theory 9 (2006), 33-45. MR2233115 (2007e:16017)

CMla, ens Cachan, CnRs, UniverSud, 61 Avenue du President Wilson, F-94230 Cachan, France

E-mail address: Patrick.LeMeur@cmla.ens-cachan.fr

Current address: Laboratoire de Mathématiques, Université Blaise Pascal \& CNRS, Complexe Scientifique Les Cézeaux, BP 80026, 63171 Aubière cedex, France

E-mail address: Patrick.LeMeur@math.univ-bpclermont.fr 